\title{
Shielding Effectiveness of Superalloy, Aluminum, and Mumetal Shielding Tapes
}

\author{
A Project Report \\ Presented to \\ The Faculty of \\ California Polytechnic State University at San Luis Obispo \\ In Partial Fulfillment \\ of the Requirements for the Degree \\ Master of Science in Aerospace Engineering \\ With Specialization in Space Systems Engineering
}

Cindy S. Cheung

April 2009

1154 W Olive Ave Apt 118

Sunnyvale, CA 94086

cinji18@hotmail.com 
(C) 2009

Cindy S. Cheung

ALL RIGHTS RESERVED 


\section{COMMITTEE MEMBERSHIP}

TITLE:

AUTHOR:

DATE SUBMITTED:

COMMITTEE CHAIR:

COMMITTEE MEMBER:

COMMITTEE MEMBER:

ADVISOR and

COMMITTEE MEMBER:
Shielding Effectiveness of Superalloy, Aluminum, and Mumetal Shielding Tapes

Cindy S. Cheung

April 2009

Dr. Jordi Puig-Sauri, Professor

Dr. Kira Abercromby, Assistant Professor

Thomas Chin, Lockheed Martin Senior Engineer 


\section{ABSTRACT \\ Shielding Effectiveness of Superalloy, Aluminum, and Mumetal Shielding Tapes Cindy S. Cheung}

Ms. Cheung performed this project as part of his Cal Poly distance-learning curriculum for a Master of Science degree in Aerospace Engineering with specialization in Space Systems Engineering. The project was performed over the Fall 2006 and Winter 2007 quarters.

Using MIL-HDBK-419A, MATLAB and Nomographs, Shielding Effectiveness for the Magnetic Field, Electric Field, and Plane Wave were calculated over a frequency range from $10 \mathrm{~Hz}$ to $1 \mathrm{GHz}$. The three shielding tapes used included superalloy, aluminum, and mumetal. Calculations for Shielding Effectiveness involve the computation of Absorption Loss, Reflection Loss, and Re-Reflection Correction Factor. From the outcome of the calculations, it was suitable to conclude that all three metals fulfill the $40 \mathrm{~dB}$ Shielding Effectiveness requirements for SGEMP fields for frequencies greater or equal to $1 \mathrm{MHz}$. Accordingly, all three shielding tapes provide at least $40 \mathrm{~dB}$ of shielding to protect certain frequencies against SGEMP Magnetic Field. However, results vary for frequencies below $1 \mathrm{MHz}$. 


\section{Acknowledgments}

I would like to acknowledge and express my gratitude for the expertise and support

that I gained during the course of this project. I wish to thank Mr. Thomas "Tom" Chin of Lockheed Martin as a senior expert in the Electromagnetic Compliance and Shielding Division for being my mentor for this project. I appreciate all of the time and efforts he spent in order to allow me to develop my understanding in the area of Electromagnetic Shielding. I would also like to thank Dr. Eric Mehiel of the Cal Poly Aerospace Engineering Department for his guidance as my academic faculty advisor. I also want to express my appreciation to Dr. Jordi Puig-Sauri and Dr. David Marshall of the Cal Poly Aerospace Engineering Department for being the other members of my project review committee. 


\section{TABLE OF CONTENTS}

LIST OF TABLES...................................................... vi

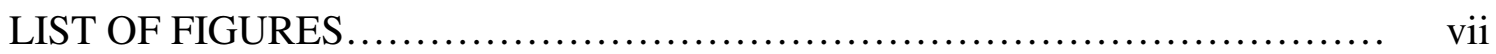

\section{CHAPTERS}

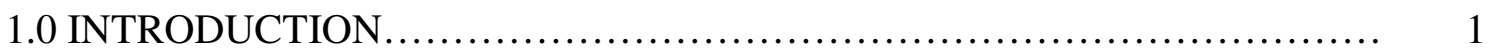

2.0 DISCUSSION AND CALCULATIONS ................................ 2

2.1 Assumptions................................................... 2

2.1.1 Shielding Tapes............................................. 2

2.1.2 Calculations............................................... 2

2.2 Shielding Effectiveness......................................... 3

2.2.1 Magnetic Field............................................. 3

2.2.2 Electric Field.............................................. 4

2.2.3 Plane Wave...................................................... 4

2.2.4 Shielding Effectiveness Calculations............................ 5

2.3 Absorption Loss................................................ 6

2.3.1 Equations................................................ 6

2.3.2 Nomographs............................................. 8

2.4 Reflection Loss.................................................... 9

2.4.1 Equations............................................... 11

2.4.2 Nomographs................................................ 13

2.5 Shielding Effectiveness when Absorption Loss $>10 \mathrm{~dB} \ldots \ldots \ldots \ldots \ldots \ldots \ldots \ldots$

2.6 Re-Reflection Correction Factor.................................. 18 
2.7 Equations vs. Nomographs...................................... 20

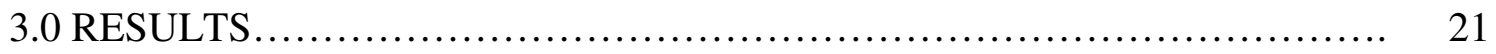

3.1 Absorption Loss.............................................. 22

3.2 Reflection Loss..................................................... 25

3.3 Shielding Effectiveness when Absorption Loss $>10 \mathrm{~dB} \ldots \ldots \ldots \ldots \ldots \ldots \ldots . . \ldots 2$

3.4 Re-Reflection Correction Factor.................................. 36

3.5 Shielding Effectiveness........................................ 40

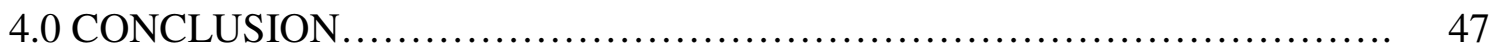

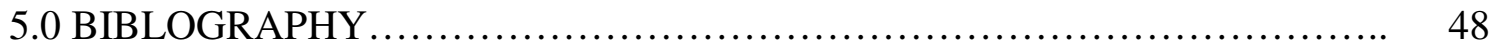

6.0 APPENDIX A-TABULATED VALUES ............................. 49

7.0 APPENDIX B-EMI SHIELDING CHARACTERISTICS OF METALS....... 52

8.0 APPENDIX C-MATLAB SOURCE CODE for Absorption Loss, Reflection Loss, Re-Reflection Correction Factor, and Shielding Effectiveness........... 53 


\section{LIST OF TABLES}

Table 1. Absorption Loss for Magnetic Field, Electric Field, and Plane Wave..... 49

Table 2. Reflection Loss for Magnetic Field, Electric Field, and Plane Wave....... 49

Table 3. Total Loss for Magnetic Field, Electric Field, and Plane Wave........... 50

Table 4. Re-Reflection Correction Factor for Magnetic Field, Electric Field, and Plane Wave..................................................... 50

Table 5. Shielding Effectiveness for Magnetic Field, Electric Field, and Plane Wave....

Table 6. EMI Shielding Characteristics of Metals............................ 52 


\section{LIST OF FIGURES}

Figure 1. Nomograph for Absorption Loss.................................................... 8

Figure 2a. Reflection Loss Nomograph for Magnetic Field..................... 13

Figure 2b. Reflection Loss Nomograph for Electric Field...................... 14

Figure 2c. Reflection Loss Nomograph for Plane Wave....................... 15

Figure 3. Absorption Loss for Magnetic Field, Electric Field, and Plane Wave produced by Matlab........................................... 22

Figure 4. Nomograph to Calculate the Absorption Loss for Magnetic Field, Electric Field, and Plane Wave...

Figure 5. Reflection Loss for Magnetic Field produced by Matlab................. 25

Figure 6. Nomograph to Calculate the Reflection Loss for Magnetic Field......... 26

Figure 7. Reflection Loss for Electric Field produced by Matlab................ 28

Figure 8. Nomograph to Calculate the Reflection Loss for Electric Field........... 29

Figure 9. Reflection Loss for Plane Wave............................... 30

Figure 10. Nomograph to Calculate the Reflection Loss for Plane Wave.......... 31

Figure 11. Total Loss for Magnetic Field................................ 33

Figure 12. Total Loss for Electric Field................................... 34

Figure 13. Total Loss for Plane Wave................................... 35

Figure 14. Re-Reflection Correction Factor for Magnetic Field................ 37

Figure 15. Re-Reflection Correction Factor for Electric Field.................. 38

Figure 16. Re-Reflection Correction Factor for Plane Wave................... 39

Figure 17a. Shielding Effectiveness for Magnetic Field...................... 40

Figure 17b. Shielding Effectiveness for Magnetic Field Up to $200 \mathrm{~dB} \ldots \ldots \ldots \ldots \ldots . . . \ldots 1$ 
Figure 18a. Shielding Effectiveness for Electric Field....................... 42

Figure 18b. Shielding Effectiveness for Electric Field Up to $200 \mathrm{~dB} \ldots \ldots \ldots \ldots \ldots \ldots .43$

Figure 19a. Shielding Effectiveness for Plane Wave....................... 44

Figure 19b. Shielding Effectiveness for Plane Wave Up to $200 \mathrm{~dB} \ldots \ldots \ldots \ldots \ldots \ldots . . . \ldots 5$ 


\subsection{INTRODUCTION}

In the aerospace industry, electromagnetic shielding plays an intricate part in the design process of any space vehicle. Electromagnetic fields from various electronic devices such as motors, batteries, and meters may have tremendous effects on each other if proper shielding protection is not appropriately implemented. It will prevent any magnetic and electric field from entering and exiting the shielded device according to requirements specified in military Military Standard Handbook MIL-HDBK-419A in the case presented. As industry standard, electromagnetic shielding is called shielding effectiveness.

Derived from Maxwell's Equations of Electromagnetic Theory, the objectives of the shielding effectiveness calculations were to determine whether or not the selected shielding tapes would conform to the $40 \mathrm{~dB}$ shielding effectiveness Requirement as indicated in EMC Specifications for magnetic field, electric field, and plane Waves from the System Generated Electro-magnetic Pulse (SGEMP). With the use of Military Standard Handbook 419A and MathWorks' MATLAB mathematical software program, the absorption loss, reflection loss, re-reflection correction factor, and the shielding effectiveness were computed for three types of shielding tape: superalloy, aluminum, and mumetal. The examined frequencies ranged from $10 \mathrm{~Hz}$ to $1 \mathrm{GHz}$. Moreover, for absorption loss and reflection loss, the results from MATLAB were also verified by nomographs, a traditional graphing method that approximates the losses. 


\subsection{DISCUSSION AND CALCULATIONS}

For the completion of these shielding calculations, references to the document, MIL-HDBK-419A, Volume I, were made in addition to the textbook, Introduction to Electromagnetic Compatibility by Clayton R. Paul.

\subsection{Assumptions}

\subsubsection{Shielding Tapes}

Shielding tapes with a thickness of $0.35 \times 10^{-3}$ inches $(889 \mu \mathrm{m})$ were placed at a distance of one meter from the electromagnetic source. The shielding tapes were assumed to be an infinite sheet, consequently eliminating edging effect.

\subsubsection{Calculations}

In order to coincide to requirements exclusively identified by the shielding equations stated in this report, some assumptions needed to be made.

First, the selected tapes were assumed to be infinite sheets of metal without geometric dependencies. As previously stated, superalloy, aluminum, and mumetal shielding tapes were selected for these calculations. The only criteria for this selection 
was each metal must have a permeability value drastically different from each other. By doing so, a range of possible shielding effectiveness values were obtainable.

Second, for the lower frequencies $(10 \mathrm{~Hz}$ to $10 \mathrm{KHz})$ of the magnetic field case, the calculated shielding range, which is roughly from $-30 \mathrm{~dB}$ to $1800 \mathrm{~dB}$, could quite possibly be impractical because geometric discrepancies exist in reality. Using these assumptions, the following quantities were calculated.

\subsection{Shielding Effectiveness}

Originating from Maxwell Equations, shielding effectiveness depicts the Faraday's principle.

\subsubsection{Magnetic Field}

In terms of magnetic field, Faraday's principle does not apply, for magnetic charges do not exist. Nevertheless, magnetic material with high permeability $(\mu>>1)$ and of ample thickness can create magnetic field attenuation by means of forming a lowreluctance path that draws the material's magnetic field.

On the other hand, thin conductive materials with low permeability also have the capability to provide shielding effectiveness for magnetic field. The shield made of the material will form an alternating magnetic field that generates eddy current on the shield 
to provide shielding effectiveness. Eddy currents produce this alternating magnetic field of opposing orientation inside the shield. As a result, as frequency increase, shielding effectiveness will increase proportionally as well.

\subsubsection{Electric Field}

Faraday's principle states that the electric field inside a conductive, spherical enclosure is nearly zero. The electric field generates both positive and negative charges which, in turn, generate a separate electric field that cancels out the original field. The thickness of the shield plays an insignificant role since electrons travel freely in conductive material.

\subsubsection{Plane Wave}

Plane wave deems the magnetic field and electric field to be completely developed, in which case:

$$
\frac{\text { MagneticField }}{\text { ElectricField }}=377 \Omega
$$

In order to achieve this condition, the distance to the radiation source needs to be far enough, or, in other words, in the Far-field region. Both the magnetic field and the electric field decrease in amplitude by $20 \mathrm{~dB}$ if the distance is increased ten times.

In the Near-field region, however, shielding effectiveness must be observed separately for magnetic field and electric field. The ratio between the fields depends on the distance from the radiation source. Magnetic field controls the Near-field when the source has low impedance; conversely, the electric field takes over when the source has 
high impedance. Moreover, when the distance to the source is $\lambda / 2 \pi$, the wave impedance converges to $377 \Omega$, and decrease linearly as the distance approach $\lambda / 2$.

\subsubsection{Shielding Effectiveness Calculations}

Shielding effectiveness indicates the capability of a given metal material to operate as protection against external electromagnetic fields and as barrier preventing internal fields from damaging other devices. Its elements consist of simply the addition of the absorption loss, reflection loss, and re-reflection correction factor:

$$
\begin{aligned}
S E_{\text {Magnetic }} & =A+R_{\text {Magetic }}-C_{\text {Magnetic }} \\
S E_{\text {Electric }} & =A+R_{\text {Electric }}-C_{\text {Electric }} \\
S E_{\text {PlaneWave }} & =A+R_{\text {PlaneWave }}-C_{\text {PlaneWave }} \\
\text { where } & \\
& \\
S E & =\text { Shielding Effectiveness } \\
A & =\text { Absorption Loss } \\
R & =\text { Reflection Loss } \\
C & =\text { Re-Reflection Correction Factor }
\end{aligned}
$$

Ultimately, the complete shielding effectiveness of a metal sheet is the summation of three factors: absorption loss, reflection loss, and re-reflection correction factor. The calculation must be applied to all three fields: electric field, magnetic field, and plane wave. Nevertheless, one should keep in mind that these calculations are only a means to predict the shielding effectiveness of the metal, and should not be considered absolute. 


\subsection{Absorption Loss}

\subsubsection{Equations}

Using the MIL-HDBK-419A as reference, the absorption loss was computed first since all three fields have identical absorption losses. The absorption loss equation is a function of the EMI Shielding Characteristic of the metal used (as shown in Appendix B) and the thickness of the tape:
$A=K_{1} l \sqrt{f \mu_{r} g_{r}}$
(in $\mathrm{dB}$ )
Eq. 2

$$
\begin{aligned}
& \text { where } \\
& \begin{aligned}
K_{1} & =131.4 \text { if } l \text { is in meters } \\
& =3.34 \text { if } l \text { is in inches } \\
l & =\text { shield thickness } \\
f & =\text { frequency } \\
\mu_{\mathrm{r}} & =\text { permeability } \\
g_{\mathrm{r}} & =\text { conductivity }
\end{aligned}
\end{aligned}
$$

The results of this equation were evaluated using Matlab, and applied to magnetic field, electric field, and plane wave. The outcome was also confirmed using nomographs.

In order to determine which shielding material is appropriate for usage, metals can be selected according to its' relative permeability and conductivity for appropriate absorption loss. Table 1 in Appendix B contains the relative EMI shielding 
characteristics, including permeability and relative conductivity for a wide range of metals. 


\subsubsection{Nomograph}

For absorption loss, a nomograph is a viable instrument for quick results. Figure 1 illustrates the nomograph for absorption loss:
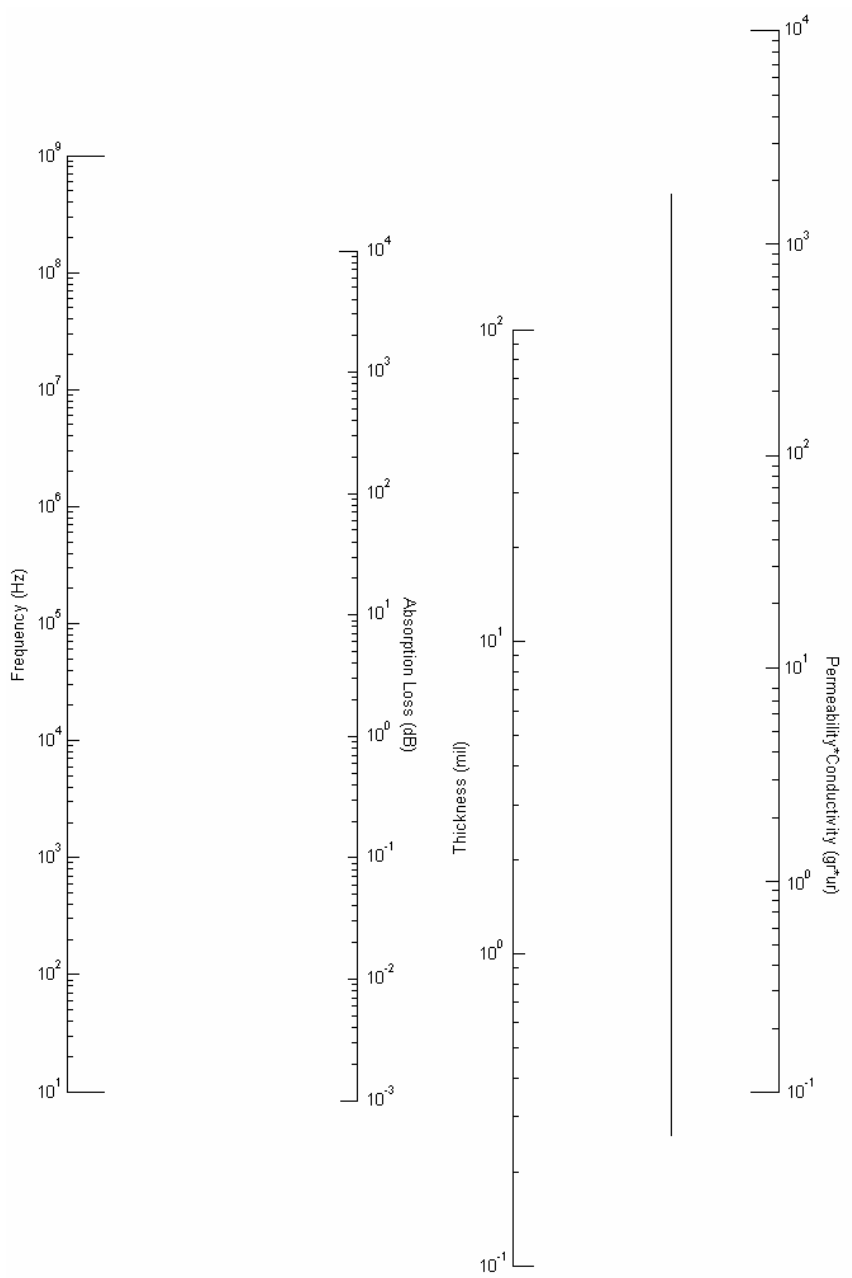

Figure 1. Nomograph for Absorption Loss 
In order to use the nomograph for absorption loss, the following steps are used:

1. Multiply the permeability and conductivity of the metal and locate the result on the scale on the right side of the nomograph.

2. Draw a line from that location to the desired thickness on the thickness scale of the nomograph. Notice that this will cross a line between the permeability-conductivity line and the thickness line. This is called the pivot line.

3. From the intersection of the pivot line and the drawn line, draw another line to the frequencies that the shield will encounter on the frequency scale on the left side of the nomograph

4. Wherever that line intersects with the absorption loss scale is the estimated absorption loss of the metal material being used.

These were the steps used in this report; however, the steps are reversible, and can be done in any necessary order should there be unknown characteristics. In this case, only the absorption loss was unknown, and the calculations were done within a range of frequencies. Therefore, rather than a single value, absorption loss had a range of values. The same applied to the Nomographs for reflection loss.

\subsection{Reflection Loss}

Reflection loss of a shield reassembles the reflection loss of a transmission line. It peaks when the impedance of the electromagnetic field is much higher or lower than impedance of the shield. When this occurs, there is an imbalance between the two impedances, and power transfers from the field to the shield to put the two in equilibrium. In cases in which reflection loss is low, metals with higher permeability and increased thickness can be utilized in order to amplify shielding effectiveness. 
In the magnetic field, the impedance of the shield and the impedance of the field are close to equilibrium at low frequencies. This produces a minimum reflection loss. As frequency increases, so does reflection loss in the magnetic field. Thus, reflection loss is nearly directly proportional to frequency.

In the electric field, the opposite is true; the higher the frequency, the closer the impedances of the shield and the field are to equilibrium, and the smaller reflection loss becomes. Hence, reflection loss is nearly inversely proportional to frequency in the electric field. 


\subsubsection{Equations}

Each field possesses separate reflection loss Equations. For magnetic field, the equation is:

$$
R_{M}=20 \log \left(\frac{C_{1}}{r \sqrt{\frac{f g_{r}}{\mu_{r}}}}+C_{2} r \sqrt{\frac{f g_{r}}{\mu_{r}}}+0.354\right)
$$

Eq. 3

where

$$
\begin{aligned}
C_{1} & =0.0117 \text { if } r \text { is in meters } \\
& =0.462 \text { if } r \text { is in inches } \\
C_{2} & =5.35 \text { if } r \text { is in meters } \\
& =0.136 \text { if } r \text { is in inches } \\
r & =\text { distance from Electromagnetic source to shield } \\
f & =\text { frequency } \\
\mu_{\mathrm{r}} & =\text { permeability } \\
g_{\mathrm{r}} & =\text { conductivity }
\end{aligned}
$$

The reflection loss equation used here is for low impedance magnetic field. This is considered near field in which $r$, the distance from the electromagnetic source, is less than the wavelength, $\lambda$, of the magnetic field divided by $2 \pi(r<\lambda / 2 \pi)$. Unlike absorption loss, which depends on shielding thickness, reflection loss depends on the distance from the electromagnetic source. 
For electric field, the equation is:

$$
R_{E}=C_{3}-10 \log \frac{\mu_{r} f^{3} r^{2}}{g_{r}}
$$

Eq. 4

$$
\begin{aligned}
& \text { where } \\
& \begin{aligned}
C_{3} & =322 \text { if } r \text { is in meters } \\
& =354 \text { if } r \text { is in inches } \\
r & =\text { distance from Electromagnetic source to shield } \\
f & =\text { frequency } \\
\mu_{\mathrm{r}} & =\text { permeability } \\
g_{\mathrm{r}} & =\text { conductivity }
\end{aligned}
\end{aligned}
$$

For the plane wave, the equation is:

$$
R_{P}=168-20 \log \sqrt{\frac{f \mu_{r}}{g_{r}}}
$$

$$
\begin{aligned}
& \text { where } \\
& \begin{array}{l}
f=\text { frequency } \\
\mu_{\mathrm{r}}=\text { permeability } \\
g_{\mathrm{r}}=\text { conductivity }
\end{array}
\end{aligned}
$$

The results were calculated using Matlab and verified by Nomographs. 


\subsubsection{Nomographs}

Figure 2a represents the nomograph for reflection Loss for magnetic field. Figure $2 \mathrm{~b}$ is for electric field, and $2 \mathrm{c}$ is for plane wave.
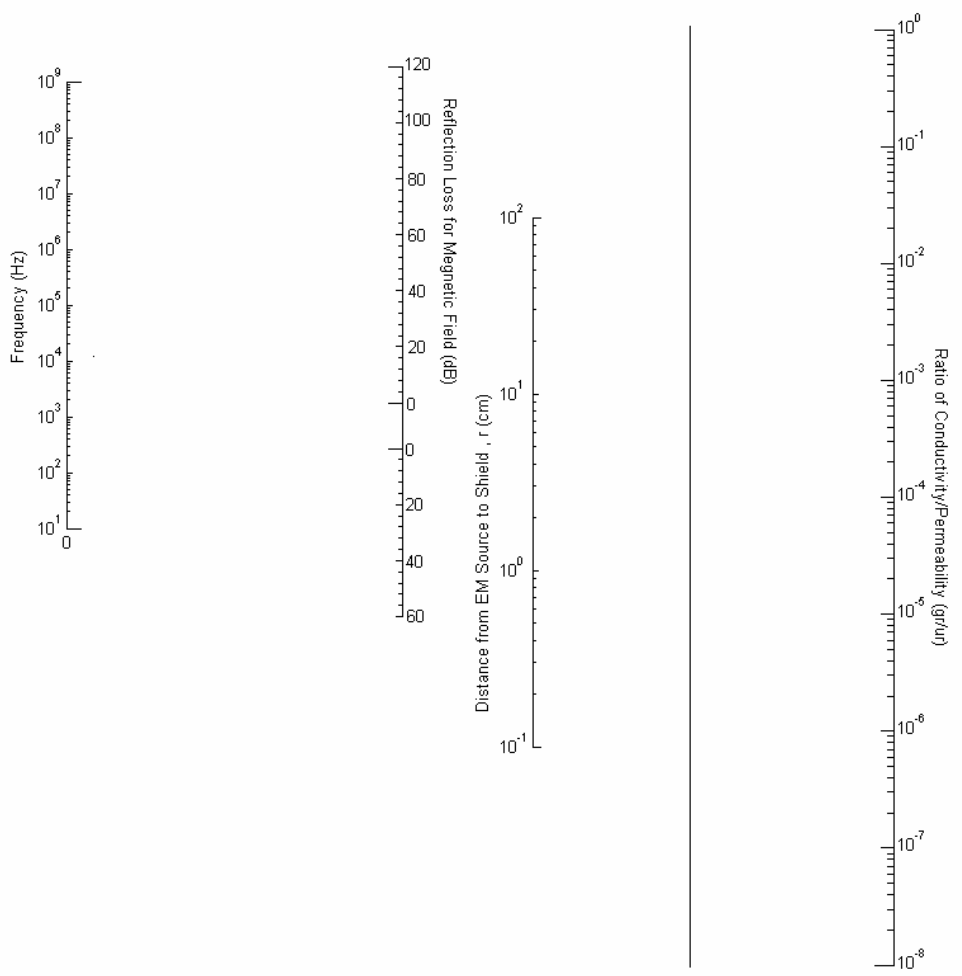

Figure 2a. Reflection Loss Nomograph for Magnetic Field 

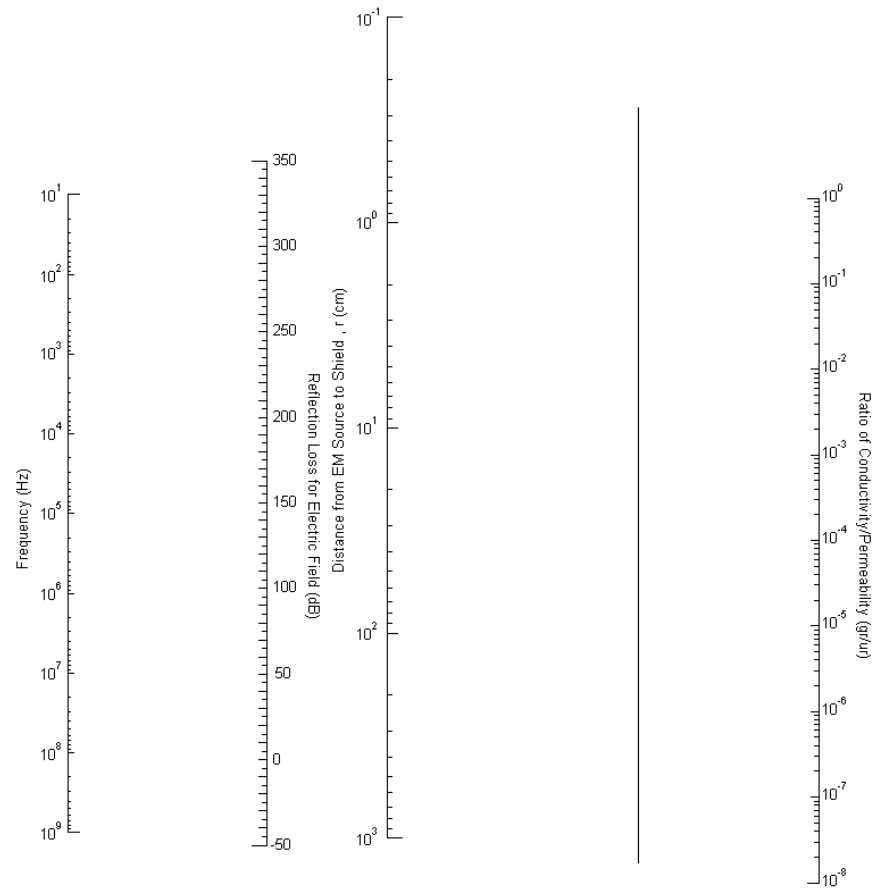

Figure 2b. Reflection Loss Nomograph for Electric Field 

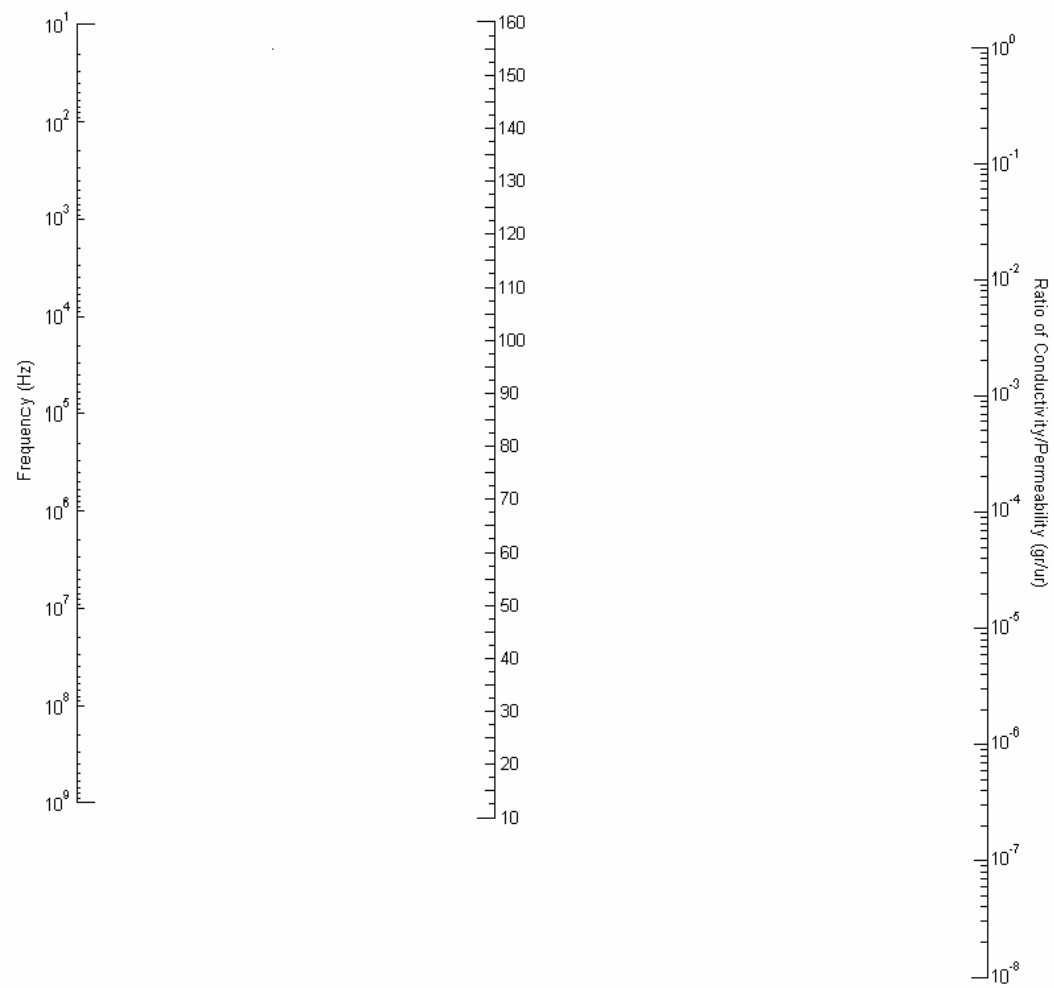

Figure 2c. Reflection Loss Nomograph for Plane Wave

The procedure for drawing graphical estimates on the nomograph for reflection loss is similar to that of absorption loss. Magnetic field and electric field have identical processes; plane wave, on the other hand, is not dependent on the distance between the electromagnetic source to the shield, and, therefore, simplifies the process:

\section{Magnetic Field and Electric Field}

1. Determine the ratio of conductivity/permeability of the metal and locate the result on the scale on the right side of the nomograph.

2. On the distance from EM source to the shield scale, pinpoint where on the scale corresponds to the distance between the EM source and the shield

3. Draw a line between the locations found on step 1 and 2. Notice that this will cross a line between the conductivity- permeability line and the distance line. This is the pivot line for this nomograph. 
4. From the intersection of the pivot line and the drawn line, draw another line to the frequencies that the shield will encounter on the frequency scale on the left side of the nomograph

5. Wherever that line intersects with the reflection loss scale is the estimated reflection loss of the metal material being used.

\section{Plane Wave}

1. Determine the ratio of conductivity/permeability of the metal and locate the result on the scale on the right side of the nomograph.

2. Draw a line from there to the frequencies that the shield will encounter on the frequency scale on the left side of the nomograph

3. Wherever that line intersects with the reflection loss scale is the estimated reflection loss of the metal material being used.

Again, these were the steps used in this report; however, the steps are reversible, and can be done in any necessary order should there be unknown characteristics. In this case, only the absorption loss was unknown, and the calculations were done within a range of frequencies. Therefore, rather than a single value, absorption loss had a range of values. The same applied to the Nomographs for reflection loss. 


\subsection{Shielding Effectiveness when Absorption Loss $>10 \mathrm{~dB}$}

For situations in which absorption loss is greater than $10 \mathrm{~dB}$, the reflected energy cannot penetrate beyond the shielding, which deems the computation of the re-reflection factor unnecessary. Hence, the total losses for shielding effectiveness in all three cases if absorption losses are greater than $10 \mathrm{~dB}$ can be calculated by summing the absorption loss and the reflection loss:

$$
\begin{aligned}
& \text { Total }_{\text {Magnetic }}=A+R_{\text {Magnetic }} \\
& \text { Total }_{\text {Electric }}=A+R_{\text {Electric }} \\
& \text { Total }_{\text {PlaneWave }}=A+R_{\text {PlaneWave }}
\end{aligned}
$$

where

$$
\begin{aligned}
A & =\text { Absorption Loss } \\
R & =\text { Reflection Loss }
\end{aligned}
$$

Note: The total loss is the shielding effectiveness if absorption loss is greater than 10 $\mathrm{dB}$.

However, from the results of the calculations as shown in the Results Section of this report, absorption loss of each metal exceeded $10 \mathrm{~dB}$ in certain frequency ranges, demonstrating the possibility of reflected energy passing through the shielding. This required the computation of the re-reflection correction factor. 


\subsection{Re-Reflection Correction Factor}

The equation for the re-reflection correction factor, $C$, is:

$$
C=20 \log \left[1-\Gamma 10^{\frac{-A}{10}}(\cos 0.23 A-j \sin 0.23 A)\right]
$$

$$
\begin{aligned}
& \text { where } \\
& \Gamma=\text { two-boundary reflection coefficient } \\
& A=\text { Absorption Loss }
\end{aligned}
$$

Each of the three fields has its' own two-boundary reflection coefficient, $\Gamma$, which is given in terms of its' own precalculation parameter, $m$. For magnetic field, the equations are:

$$
\begin{aligned}
& \Gamma=4 \frac{\left(1-m^{2}\right)^{2}-2 m^{2}+j 2 \sqrt{2} m\left(1-m^{2}\right)}{\left[(1+\sqrt{2} m)^{2}+1\right]^{2}} \\
& m=\frac{4.7 \times 10^{-2}}{r} \sqrt{\frac{\mu_{r}}{f g_{r}}}
\end{aligned}
$$

Eq. 8

where

$$
\begin{aligned}
& r=\text { distance from Electromagnetic source to shield } \\
& f=\text { frequency } \\
& \mu_{\mathrm{r}}=\text { permeability } \\
& g_{\mathrm{r}}=\text { conductivity }
\end{aligned}
$$


For the electric field, the equations are:

$$
\begin{aligned}
& \Gamma=4 \frac{\left(1-m^{2}\right)^{2}-2 m^{2}-j 2 \sqrt{2} m\left(1-m^{2}\right)}{\left[(1-\sqrt{2} m)^{2}+1\right]^{2}} \\
& m=0.205 \times 10^{-16} r \sqrt{\frac{\mu_{r} f^{3}}{g_{r}}}
\end{aligned}
$$

where

$$
\begin{aligned}
& r=\text { distance from Electromagnetic source to shield } \\
& f=\text { frequency } \\
& \mu_{\mathrm{r}}=\text { permeability } \\
& g_{\mathrm{r}}=\text { conductivity }
\end{aligned}
$$

For the plane wave, the equations are:

$$
\begin{aligned}
& \Gamma=4 \frac{\left(1-m^{2}\right)^{2}-2 m^{2}-j 2 \sqrt{2} m\left(1-m^{2}\right)}{\left[(1+\sqrt{2} m)^{2}+1\right]^{2}} \cong 1 \\
& m=9.77 \times 10^{-10} \sqrt{\frac{f \mu_{r}}{g_{r}}}
\end{aligned}
$$

where

$$
\begin{aligned}
& r=\text { distance from Electromagnetic source to shield } \\
& f=\text { frequency } \\
& \mu_{\mathrm{r}}=\text { permeability } \\
& g_{\mathrm{r}}=\text { conductivity }
\end{aligned}
$$


Using the proper two-boundary reflection coefficient, $\Gamma$, and its' precalculation parameter, $m$, the appropriate corresponding re-reflection correction Factors for each case were calculated in order to adjust shielding effectiveness accurately.

As established earlier, the re-reflection correction factor is necessary for absorption losses less than $10 \mathrm{~dB}$ in order to prevent reflected energy from penetrating beyond the shielding. This factor can be either positive or negative if the shield is very thin.

\subsection{Equations vs. Nomographs}

As shown, shielding effectiveness equations can be quite problematic and timeconsuming without the use of a computational software such as MATLAB. Hence, for rapid results, Nomographs can be used with minimal inaccuracies when available. 


\subsection{RESULTS}

Because of the large quantity of calculations, the probability of computational errors is fairly high; therefore, a software computational script was created using MathWorks' MATLAB program.

For all of the figures in the Results Sections, superalloy is indicated in blue, aluminum in green, and mumetal in red. 


\subsection{Absorption Loss}

The following absorption losses produced by Matlab after inputting the established initial conditions. This figure applied to all three fields being the magnetic field, electric field, as well as plane wave:

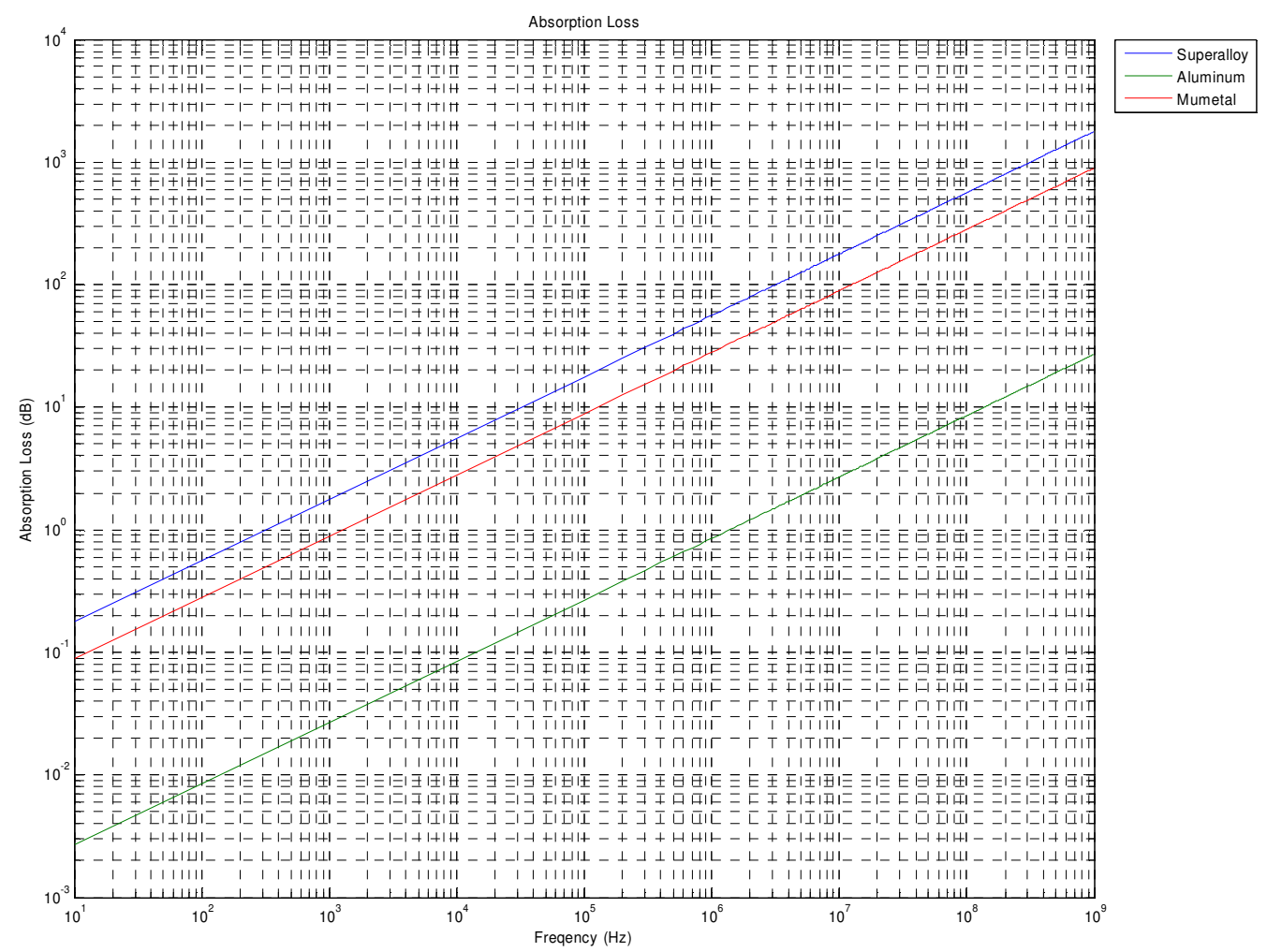

Figure 3. Absorption Loss for Magnetic Field, Electric Field, and Plane Wave produced by Matlab

Similar findings were confirmed by a nomograph. As a reminder, because

Nomographs are handdrawn, it is only appropriate to use it as an approximation and not taken as the ultimate answer. 


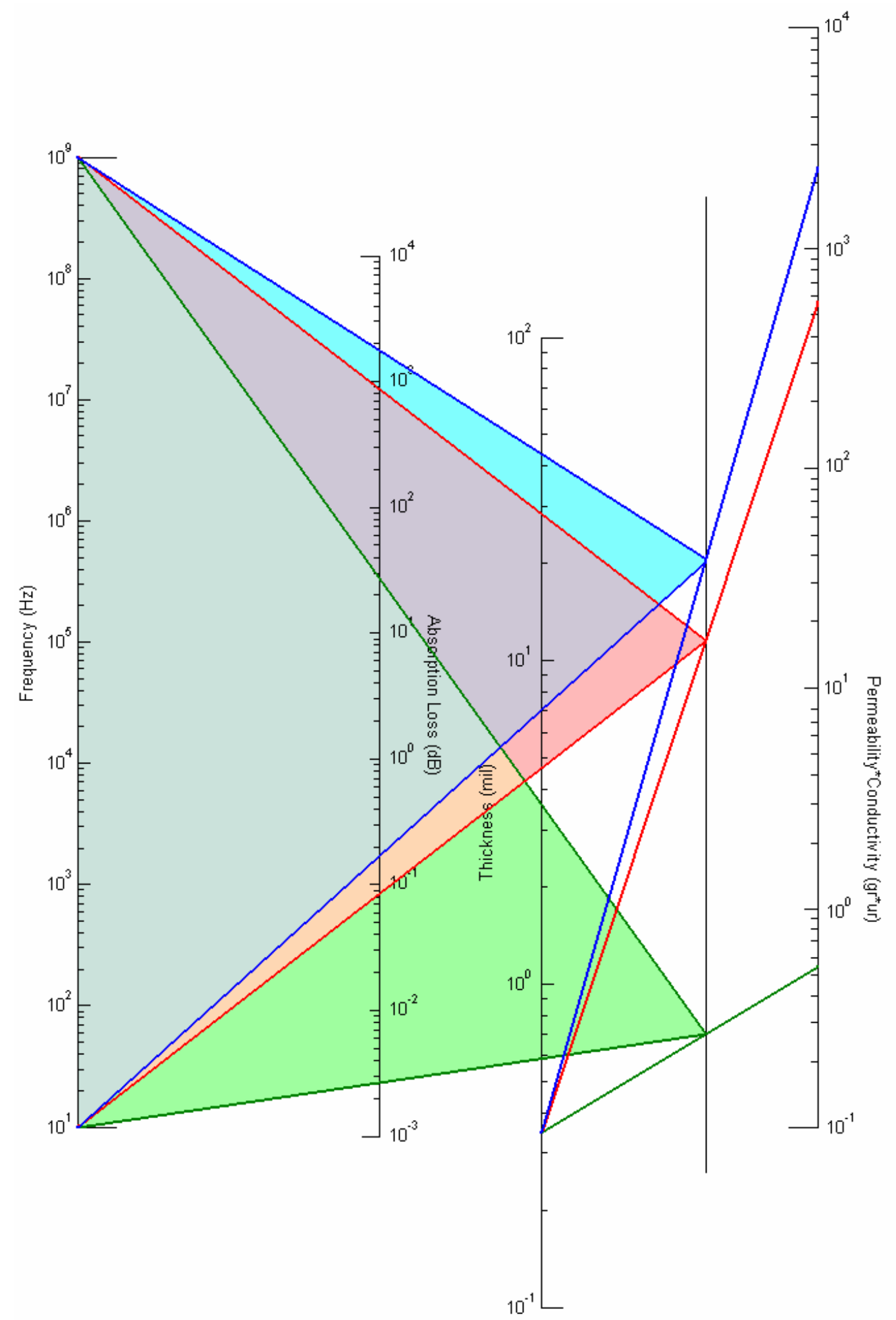

Figure 4. Nomograph to Calculate the Absorption Loss for Magnetic Field, Electric Field, and Plane Wave 
With close examination of both Figure 3 and 4, it was confirmed that a) the mathematical equations and the nomograph result in identical conclusions and can be done independently, and b) the calculated absorption losses where correct and accurate. In addition, both figures show that aluminum shielding tape had the least absorption loss, making it he most vulnerable to reflected energy. Thus, although it was evident that all three materials would require the use of the re-reflection correction factor, aluminum in particular would rely on this facter for the widest range of frequencies. Further confirmation could be seen in Table 1, which was produced by Microsoft Excel and Matlab, in Appendix A section of this report. 


\subsection{Reflection Loss}

The following graphs shows the reflection losses for the magnetic field, electric

field, and plane wave, respectively, produced by Matlab along with the corresponding Nomographs:

\section{Magnetic Field}

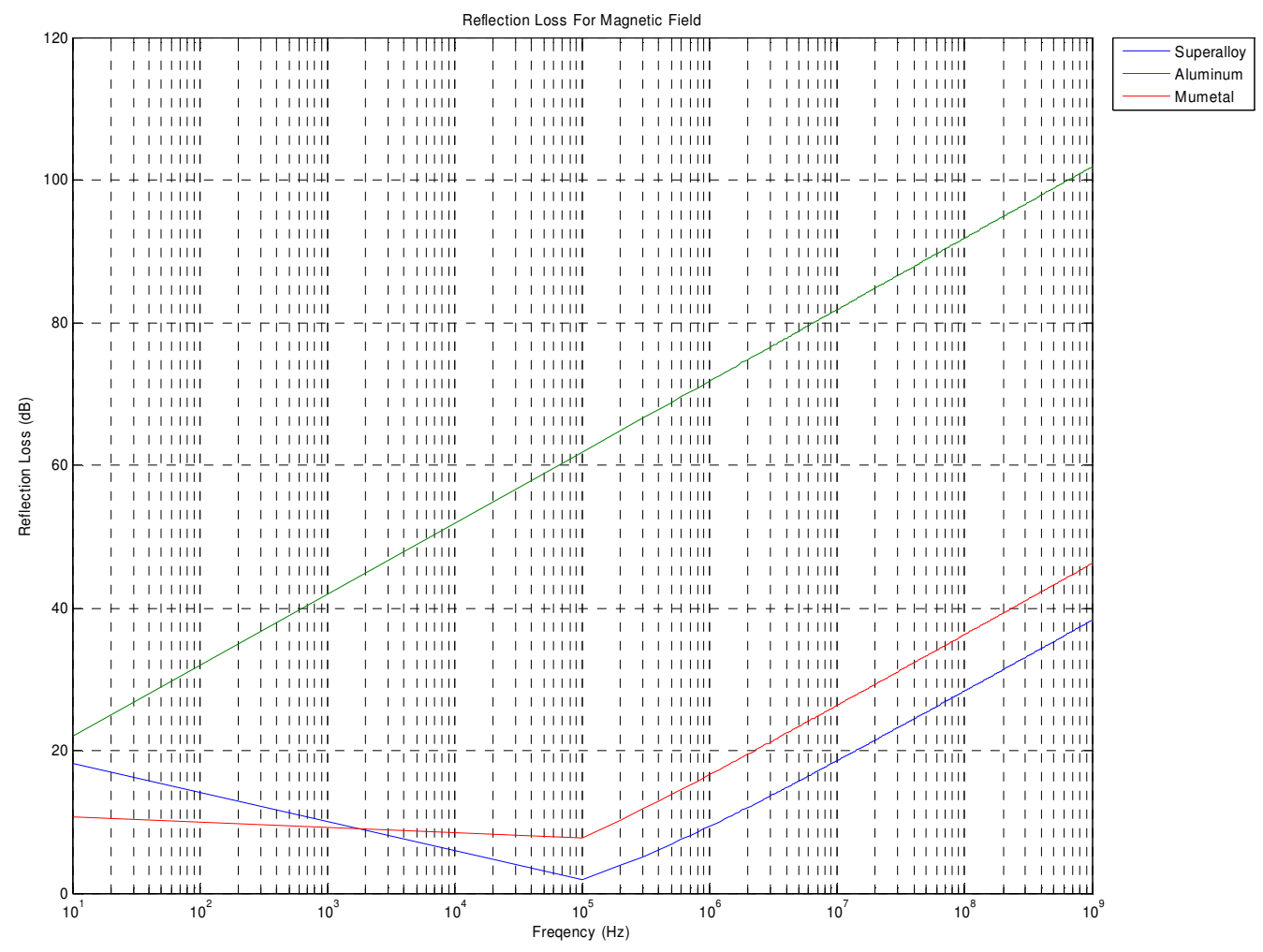

Figure 5. Reflection Loss for Magnetic Field produced by Matlab

The results of Figure 5 could be verified by the nomograph in Figure 6: 


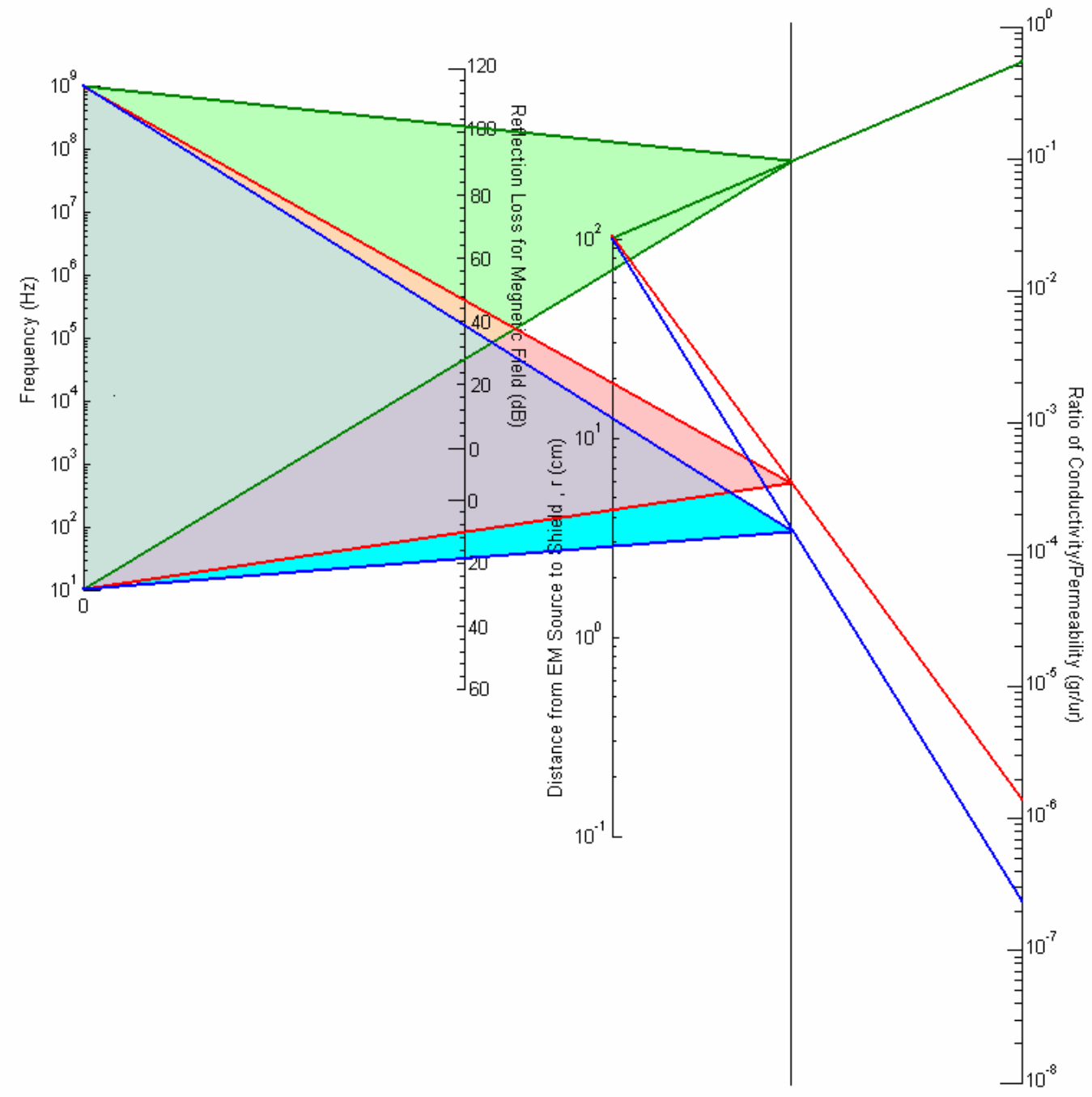

Figure 6. Nomograph to Calculate the Reflection Loss for Magnetic Field 
With close examination of both Figure 5 and 6, aluminum demonstrated the most reflection loss in the magnetic field while superalloy and mumetal projected similar levels even though their relative permeability values are greatly different. Further confirmation could be seen in Table 2, which was produced by Microsoft Excel and Matlab, in the Appendix A section of this memorandum. 


\section{Electric Field}

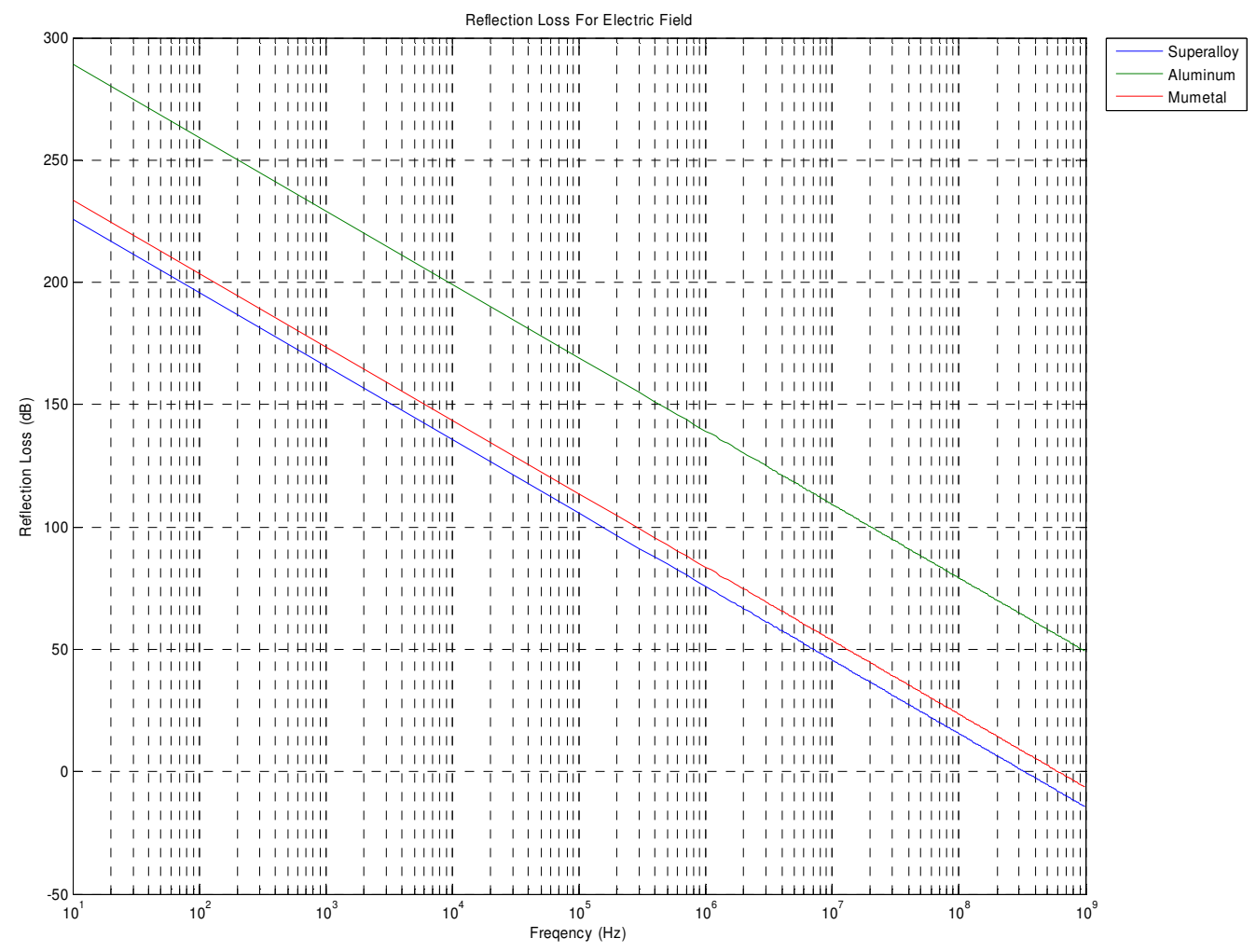

Figure 7. Reflection Loss for Electric Field produced by Matlab 
The results of Figure 7 could be verified by the nomograph in Figure 8:

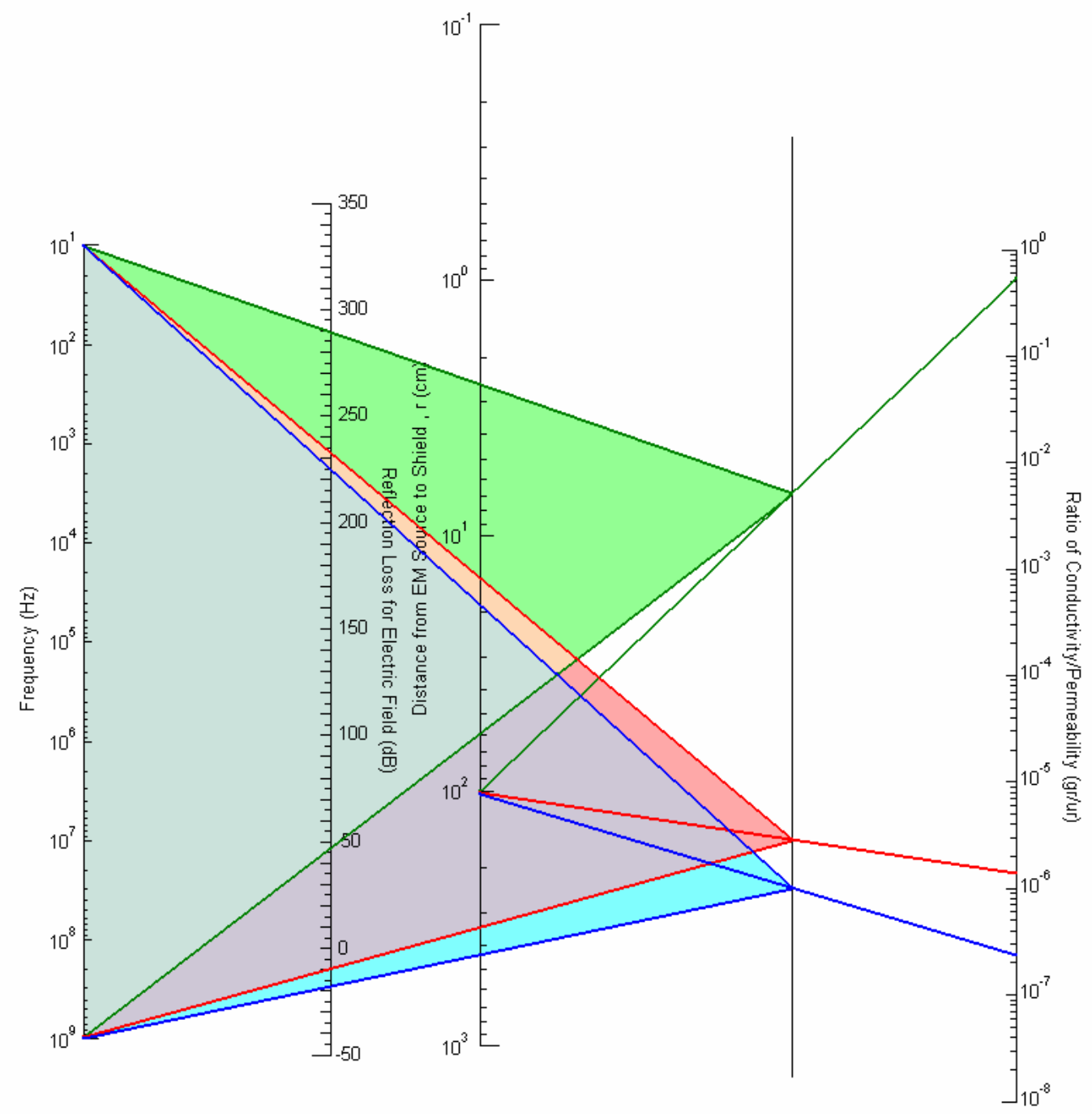

Figure 8. Nomograph to Calculate the reflection Loss for Electric Field 
Similarly, Figures 7 and 8 shows that aluminum demonstrated the most reflection loss in the electric field as well while superalloy and mumetal projected levels close to each other. Further confirmation could be seen in Table 2, which was produced by Microsoft Excel and Matlab, in the Appendix A section of this report.

\section{Plane Wave}

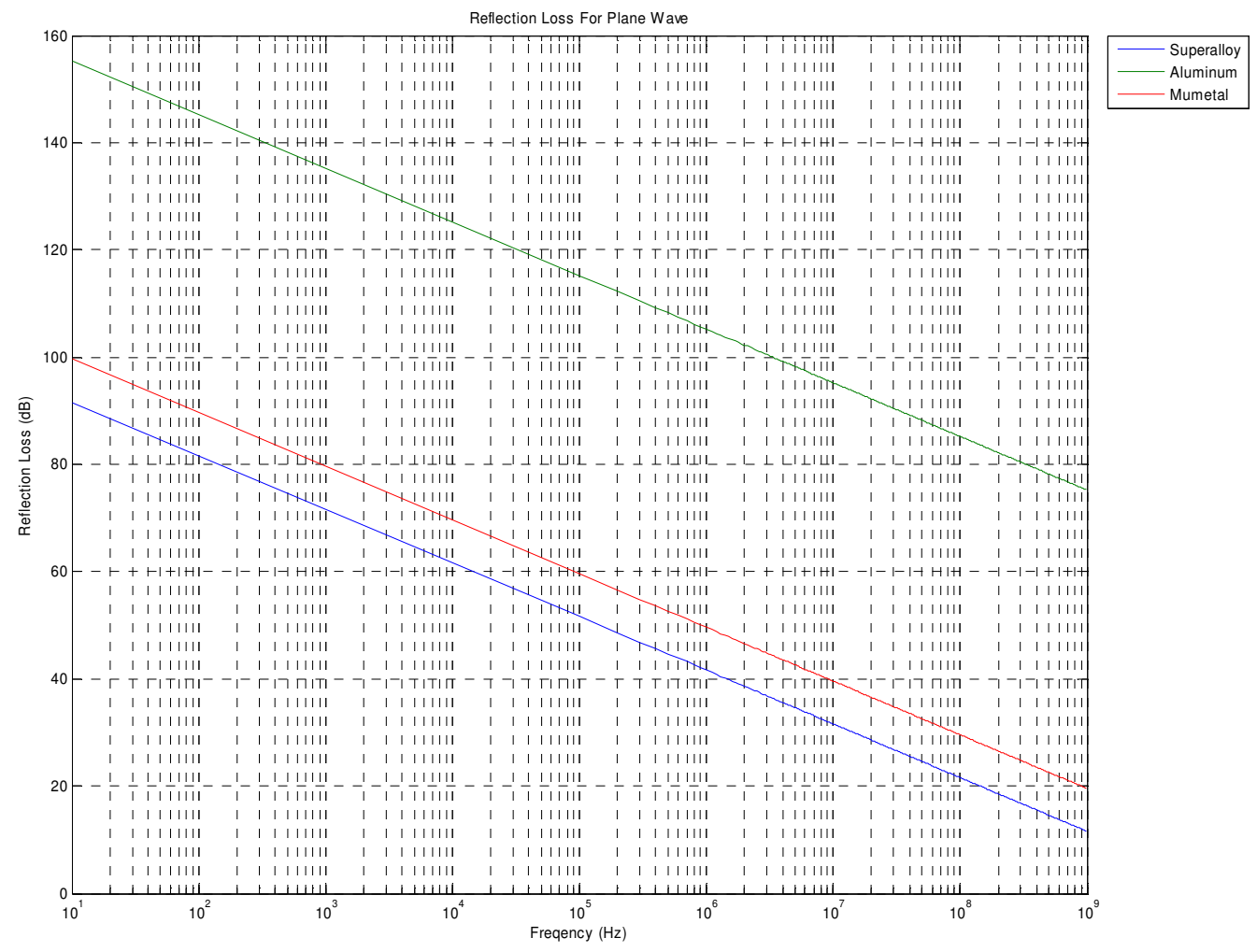

Figure 9. Reflection Loss for Plane Wave 
The results of Figure 9 could be verified by the nomograph in Figure 10:

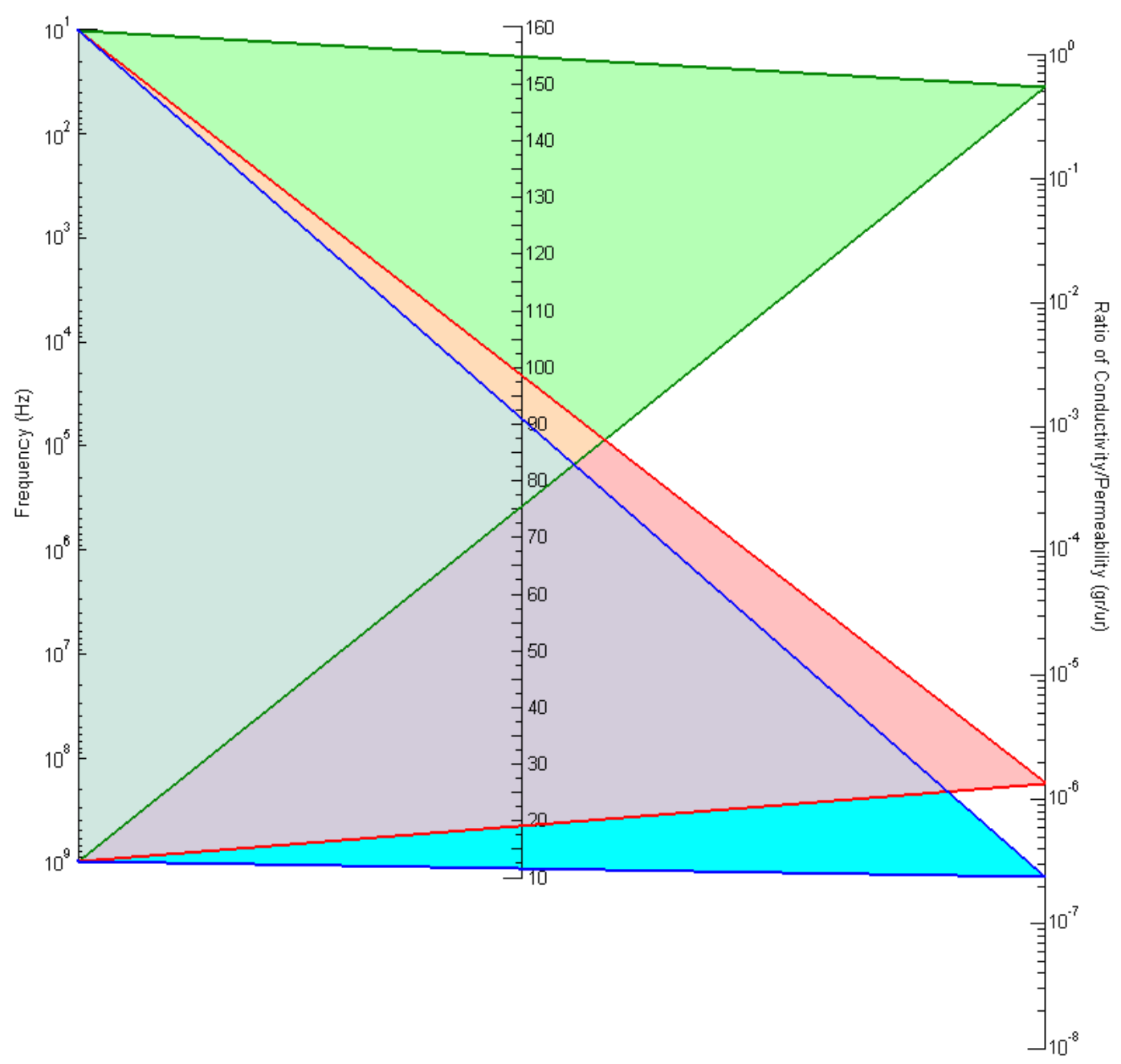

Figure 10. Nomograph to Calculate the Reflection Loss for plane Wave

Again, Figure 9 and 10, aluminum demonstrated the most reflection loss in the plane wave while superalloy and mumetal projected similar levels. Further confirmation could be seen in Table 2, which was produced by Microsoft Excel and Matlab, in the Appendix A section of this report. 


\subsection{Shielding Effectiveness when Absorption Loss $>10 \mathrm{~dB}$}

These are the resulting shielding effectiveness graphs produced by Matlab for cases in which absorption losses are greater than $10 \mathrm{~dB}$. Again, this is the sum of the absorption loss and the reflection loss. The re-reflection correction factor is unnecessary since the addition of the factor will not greatly hinder shielding effectiveness results.

Because Nomographs cannot be used to estimate the re-reflection correction factor, their usage was eliminated from here on out. 


\section{Magnetic Field}

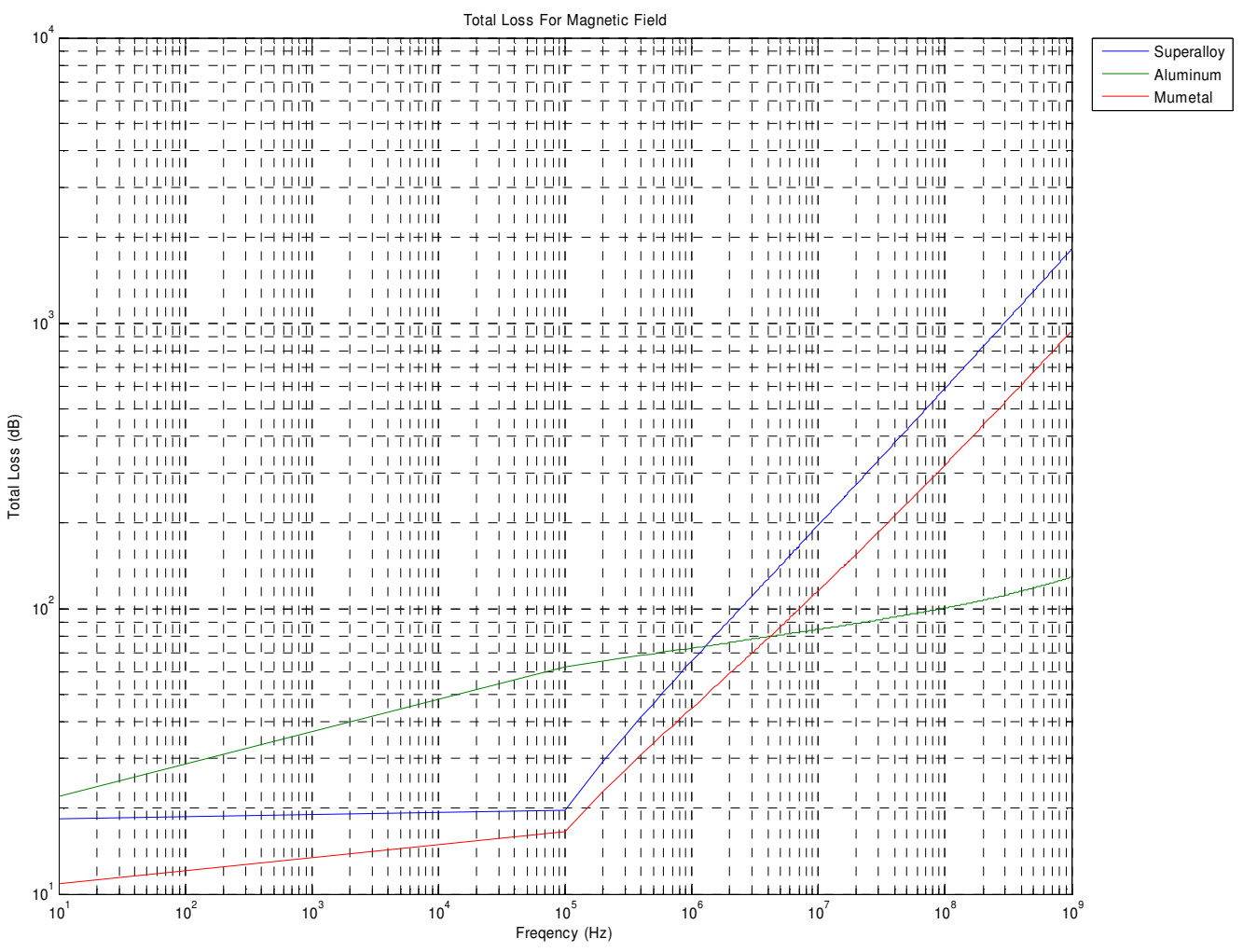

Figure 11. Total Loss for Magnetic Field 


\section{Electric Field}

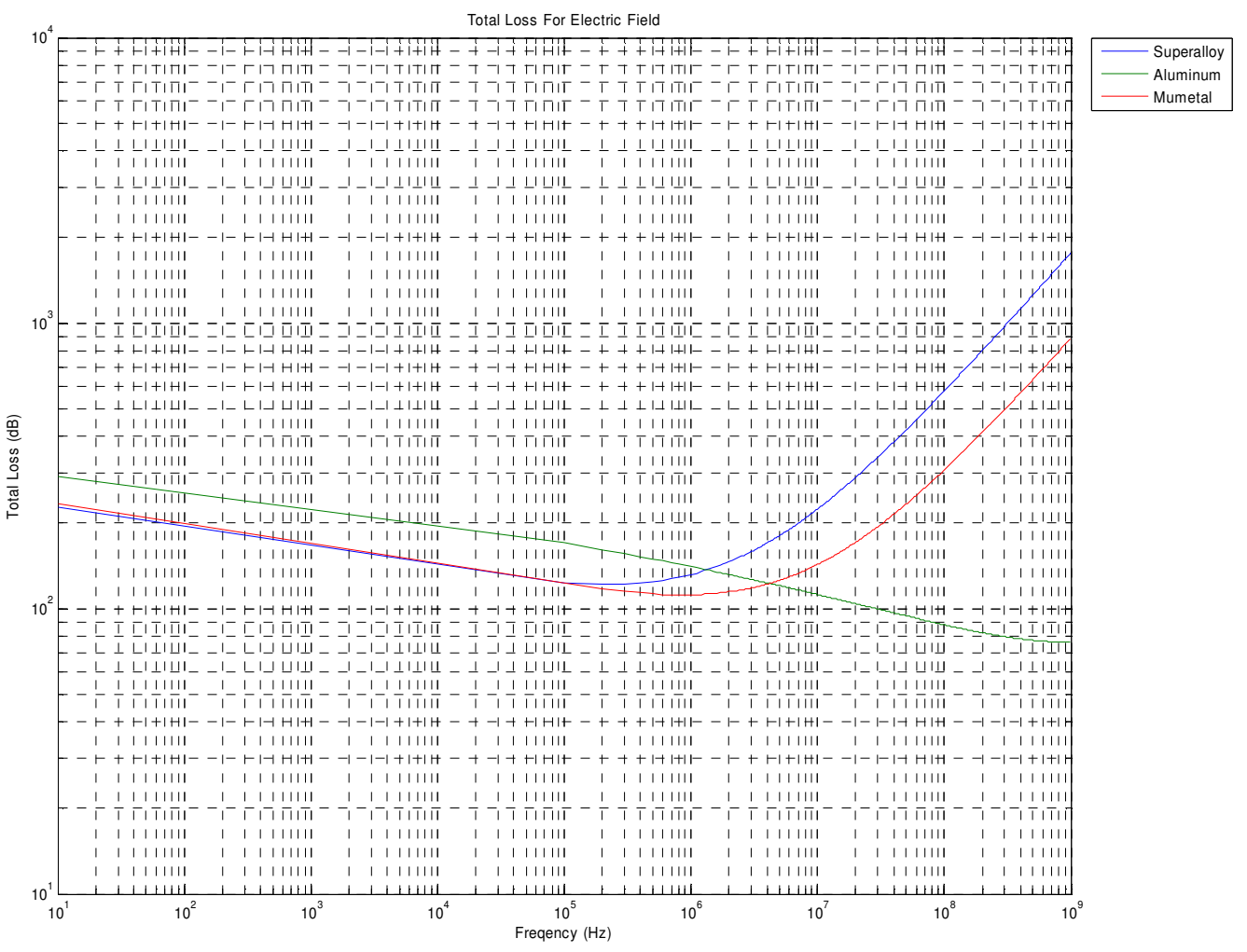

Figure 12. Total Loss for Electric Field 


\section{Plane Wave}

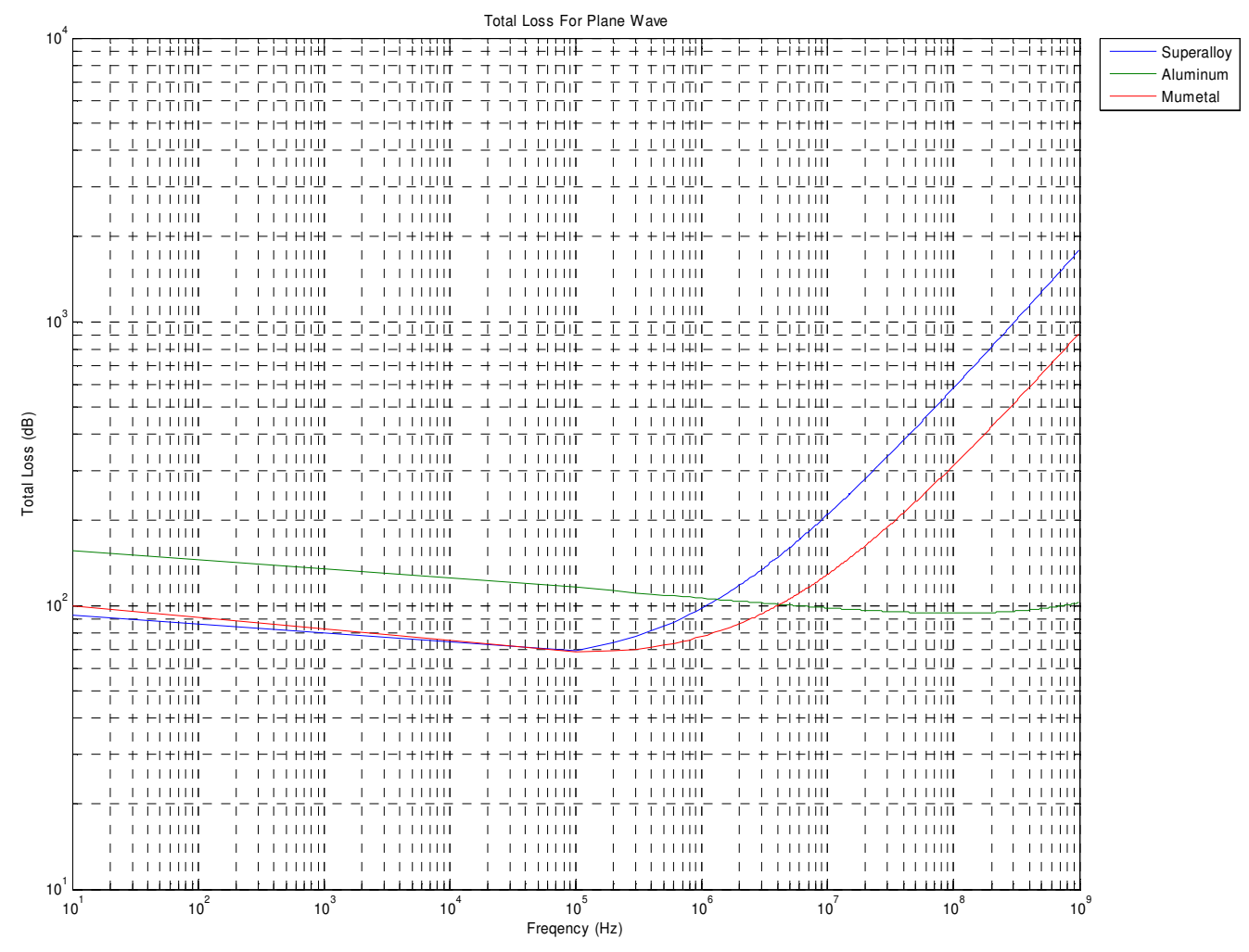

Figure 13. Total Loss for Plane Wave

For all three fields, aluminum had the shortest range of shielding effectiveness, from approximatedly $20 \mathrm{~dB}$ to $150 \mathrm{~dB}$, in the frequency range of $10 \mathrm{~Hz}$ to $1 \mathrm{GHz}$. From $10 \mathrm{~Hz}$ to $1 \mathrm{MHz}$, aluminum had greater shielding effectiveness because of greater reflection loss as opposed to superalloy and mumetal. Nevertheless, for frequencies grater than $1 \mathrm{MHz}$, the absorption loss of superalloy and mumetal surpassed that of aluminum, and as a result, exceeded the shielding effectiveness of aluminum. One must keep in mind, however, that at this point in the calculations, only shielding effectiveness with an absorption loss of less than $10 \mathrm{~dB}$ could be considered accurate as the rereflection correction factor had been excluded thus far. 
Tabulated results of shielding effectiveness without the re-reflection correction factor could be observed in Table 3 of the Appendix A section.

\subsection{Re-Reflection Correction Factor}

Since a large portion of the absorption loss results exceeded $10 \mathrm{~dB}$, calculations of the re-reflection correction factor were required for proper shielding effectiveness results. The following graphs represent the re-reflection correction factor for each of the three situations. 


\section{Magnetic Field}

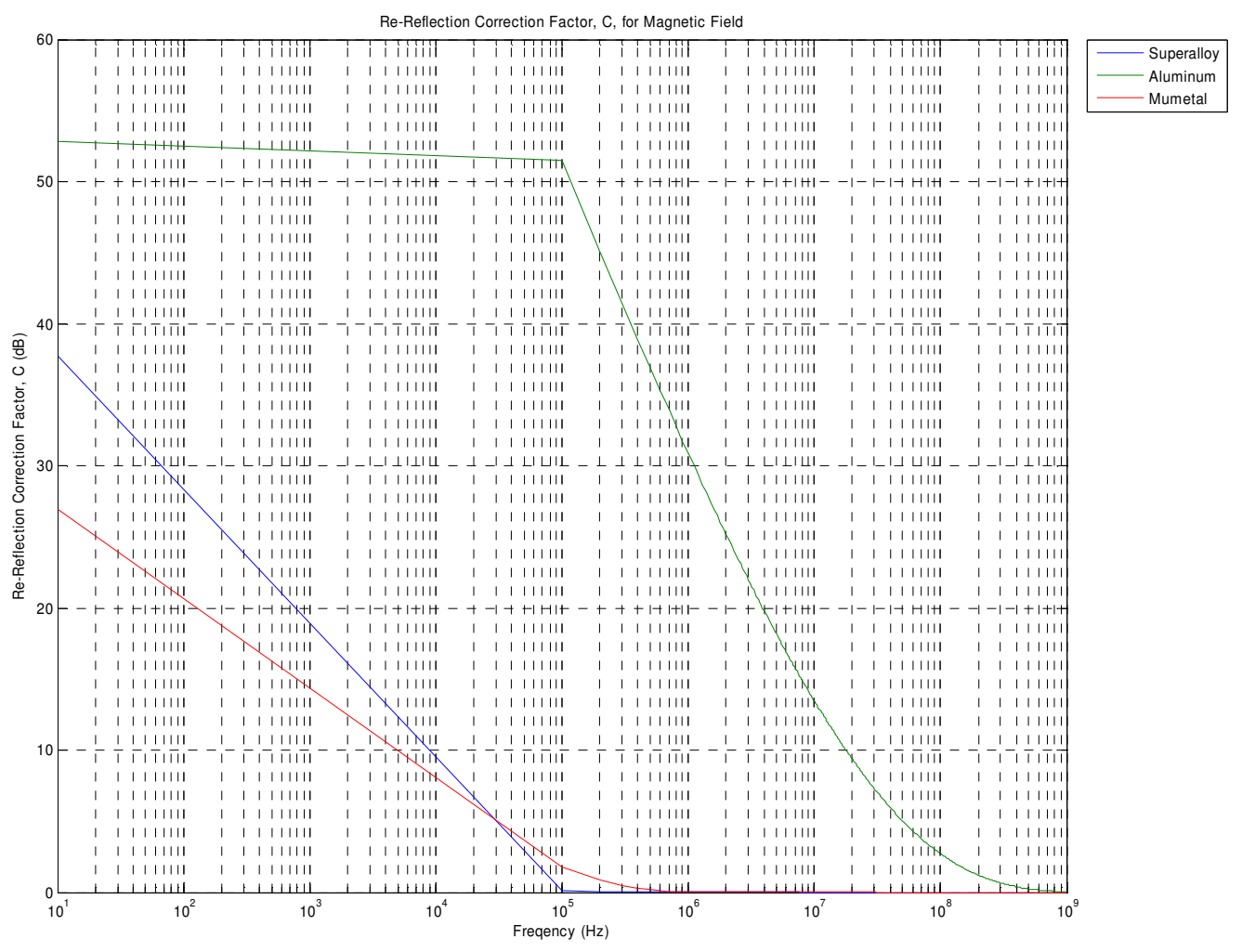

Figure 14. Re-Reflection Correction Factor for Magnetic Field 


\section{Electric Field}

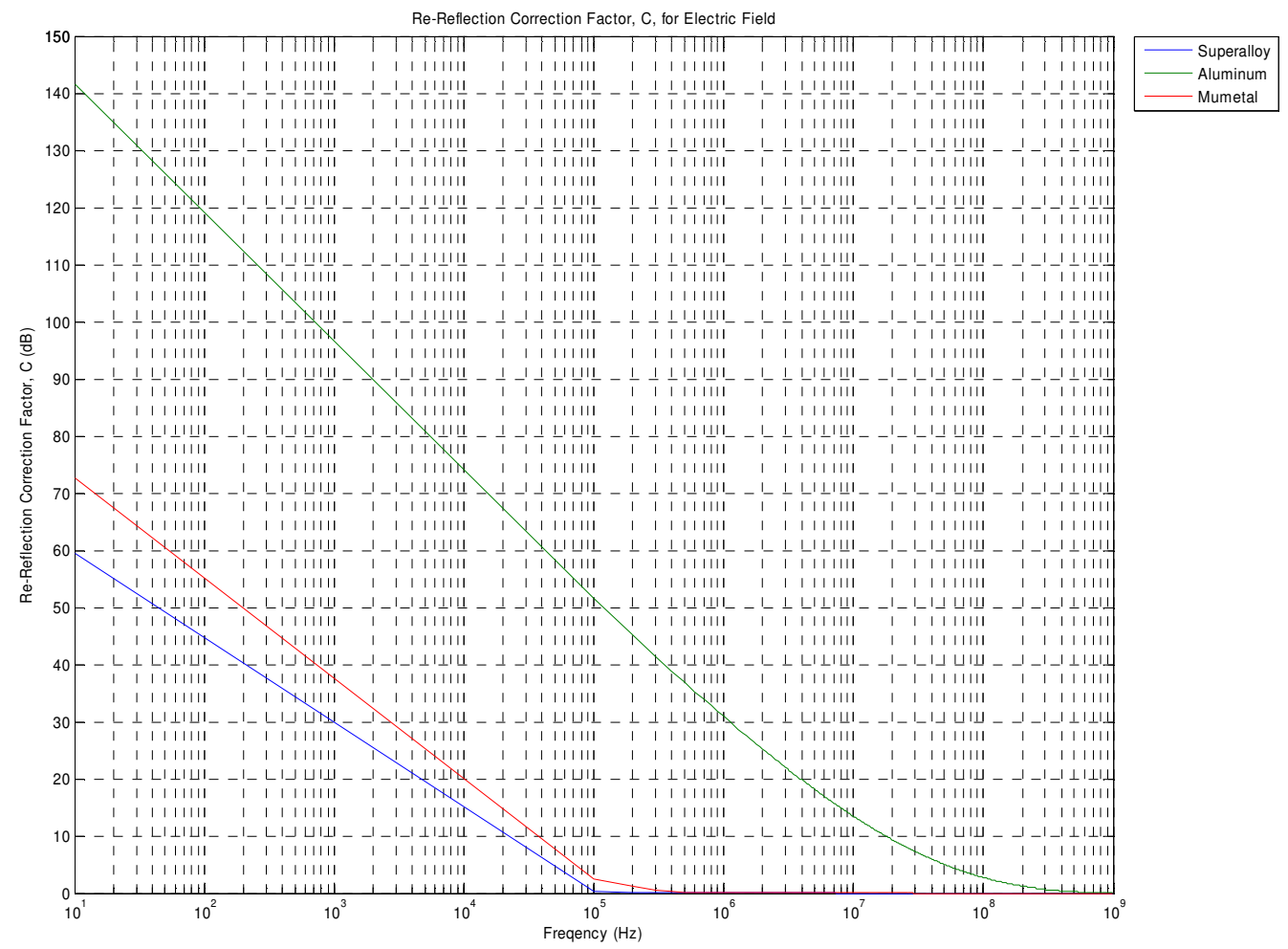

Figure 15. Re-Reflection Correction Factor for Electric Field 


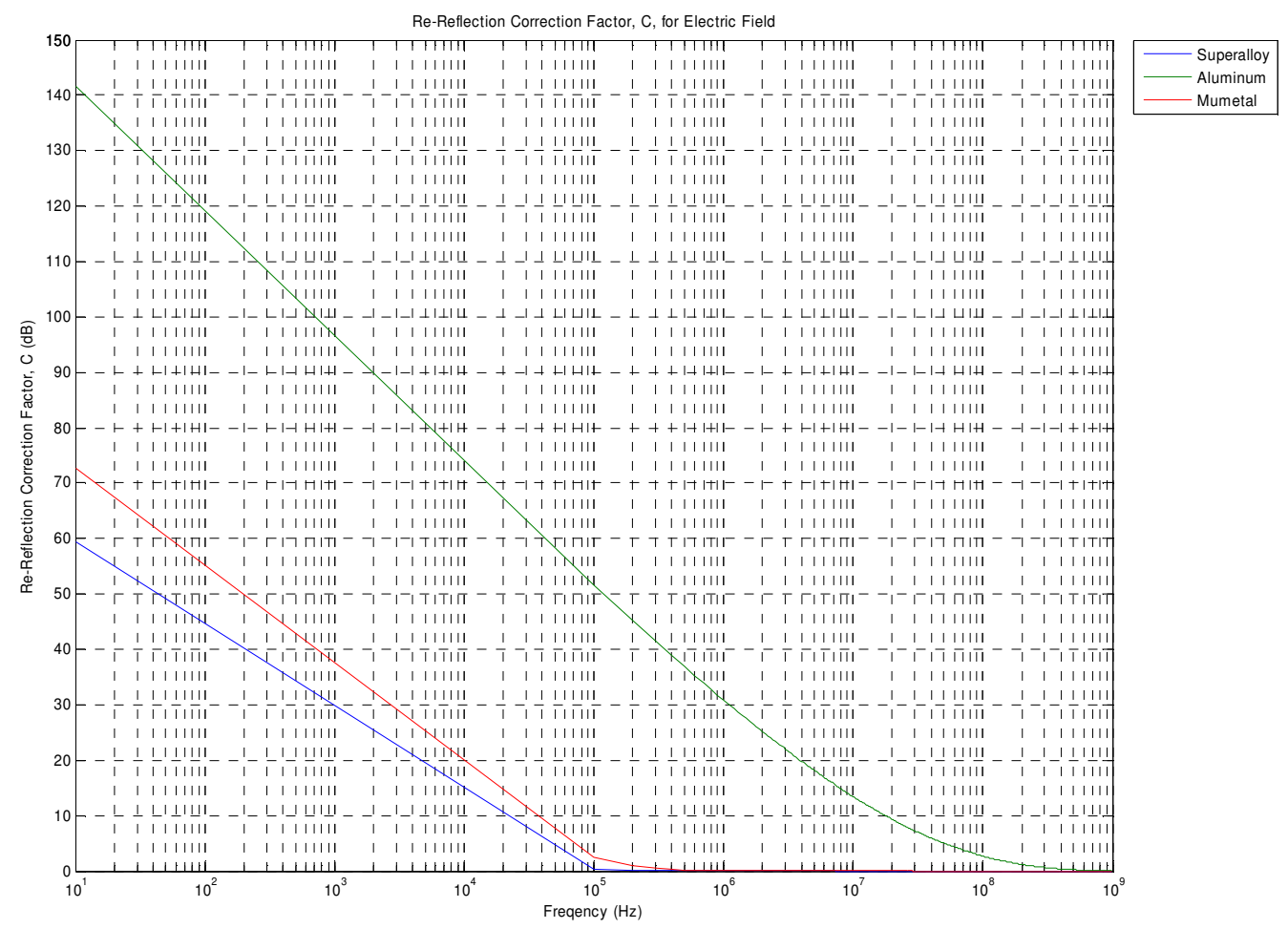

Figure 16. Re-Reflection Correction Factor for Plane Wave

Tabulated results of re-reflection correction factor could by observed in Table 4 of the Appendix A section.

When Figure 14, 15, and 16 where examined along with Figure 3, it was evident that the re-reflection correction factor was necessary only when absorption losses were less than $10 \mathrm{~dB}$. Referring back to Figure 3, absorption loss for aluminum did not pass beyond $10 \mathrm{~dB}$ until approximately $100 \mathrm{MHz}$, and for superalloy and mumetal, between $10 \mathrm{KHz}$ and $100 \mathrm{KHz}$. This directly corresponds to the re-reflection correction factor figures. In all three of the latter figures, the factor approached zero when frequency reached $100 \mathrm{MHz}$ for aluminum and $100 \mathrm{KHz}$ for superalloy and mumetal. Therefore, 
this confirmed the unessential computation of the re-reflection correction factor when absorption loss is greater than $10 \mathrm{~dB}$.

\subsection{Shielding Effectiveness}

These figures represent the shielding effectiveness from Matlab with the use of the re-reflection correction factor, for each case.

\section{Magnetic Field}

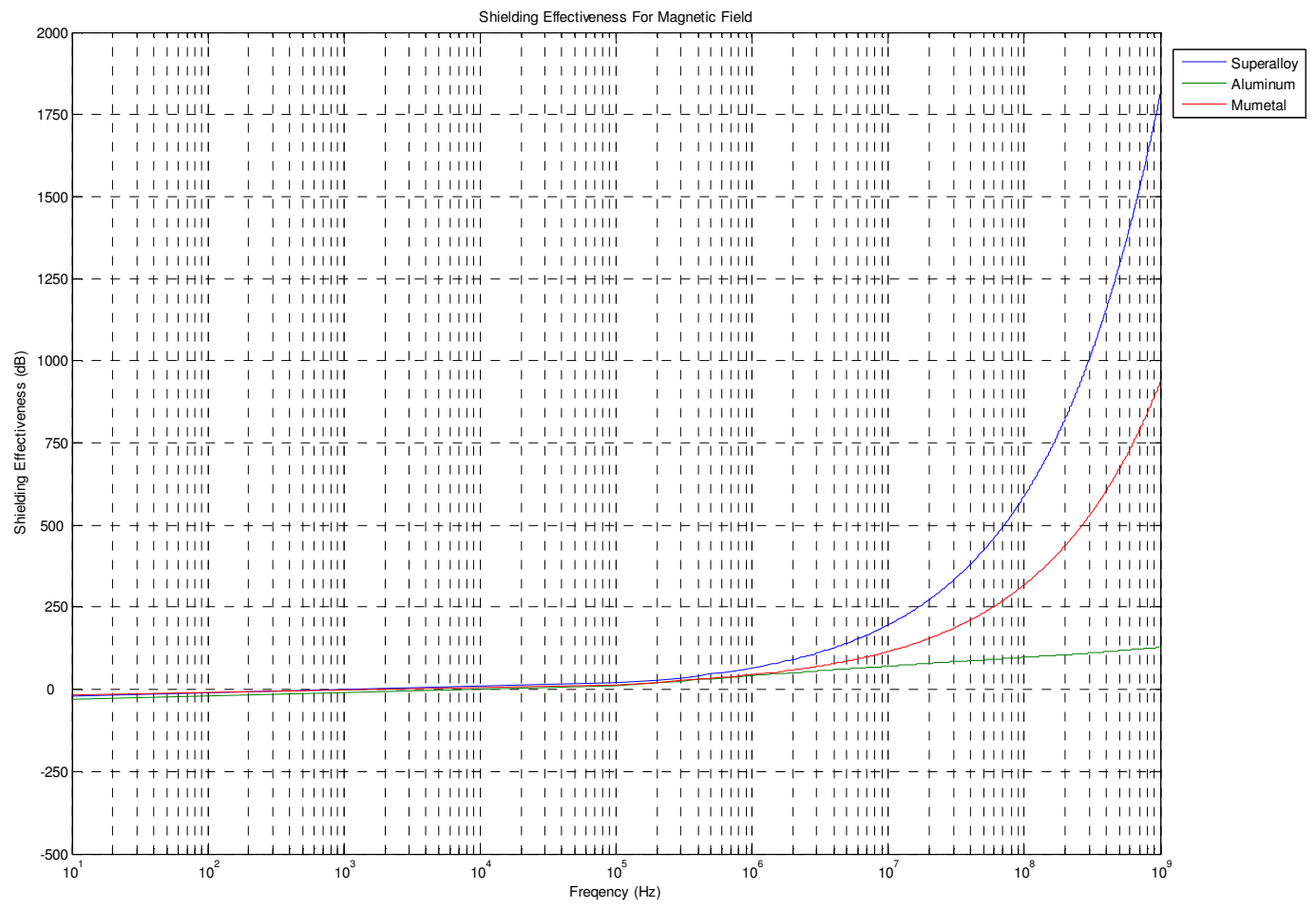

Figure 17a. Shielding Effectiveness for Magnetic Field 


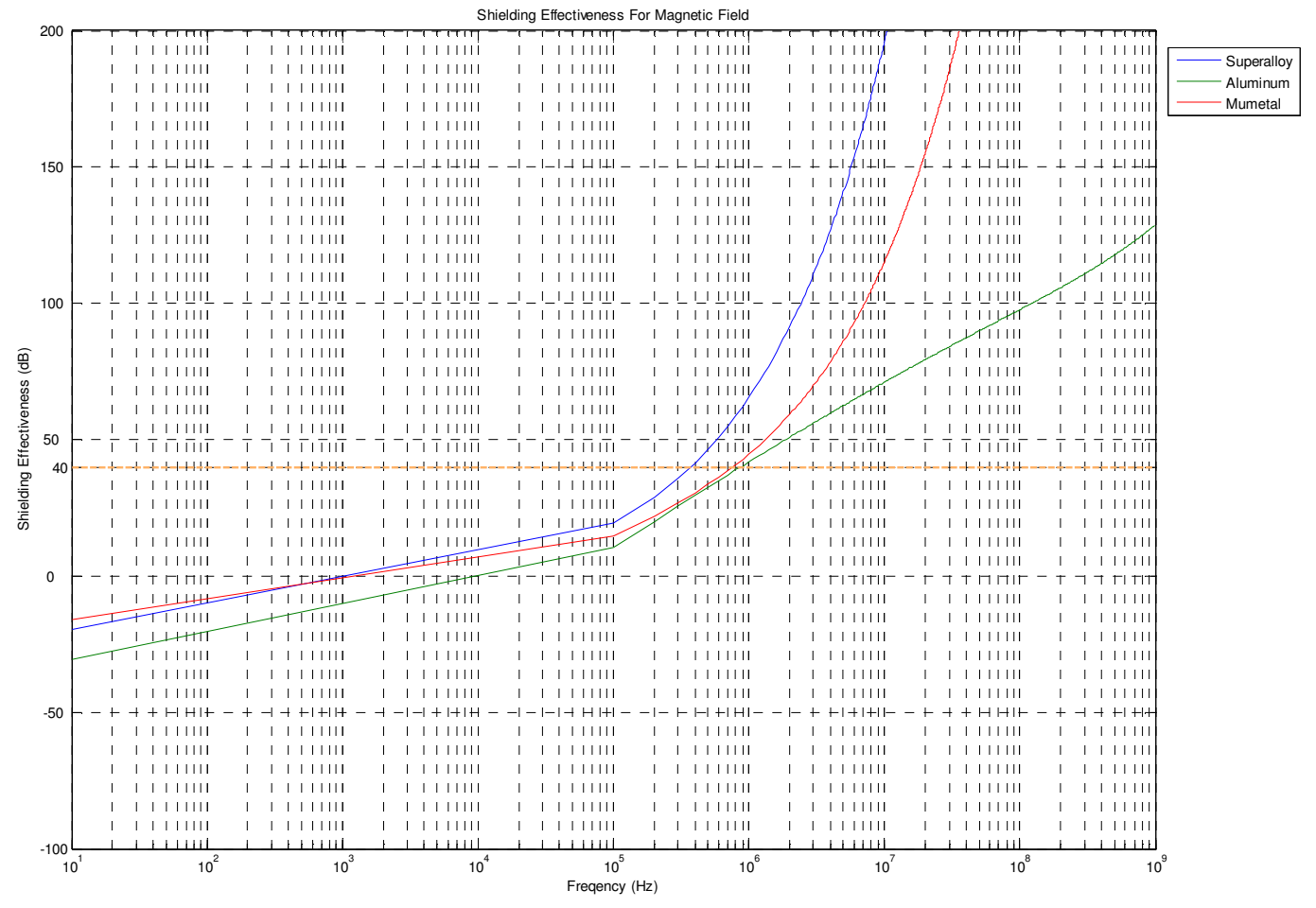

Figure 17b. Shielding Effectiveness for Magnetic Field Up to $200 \mathrm{~dB}$ 


\section{Electric Field}

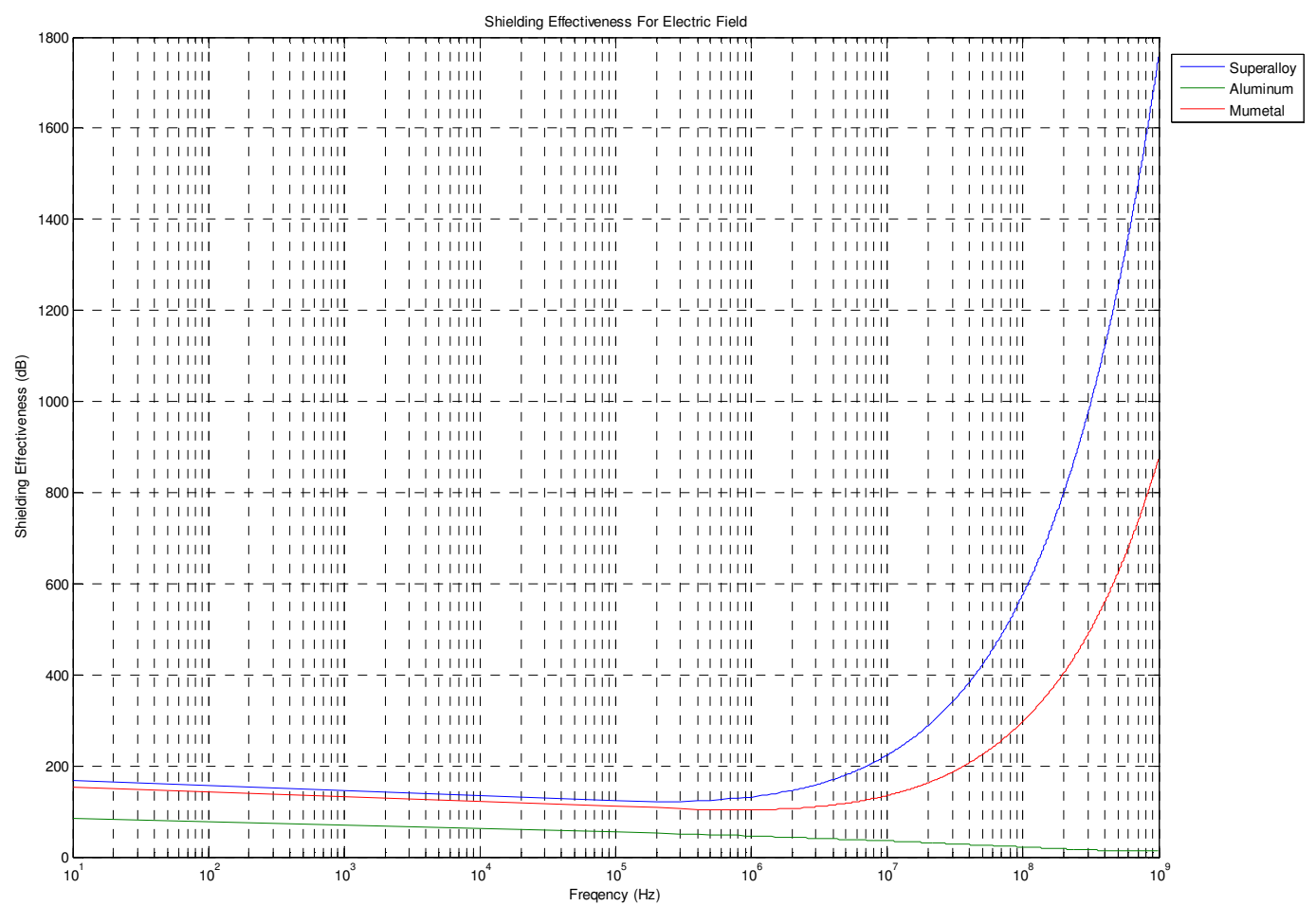

Figure 18a. Shielding Effectiveness for Electric Field 


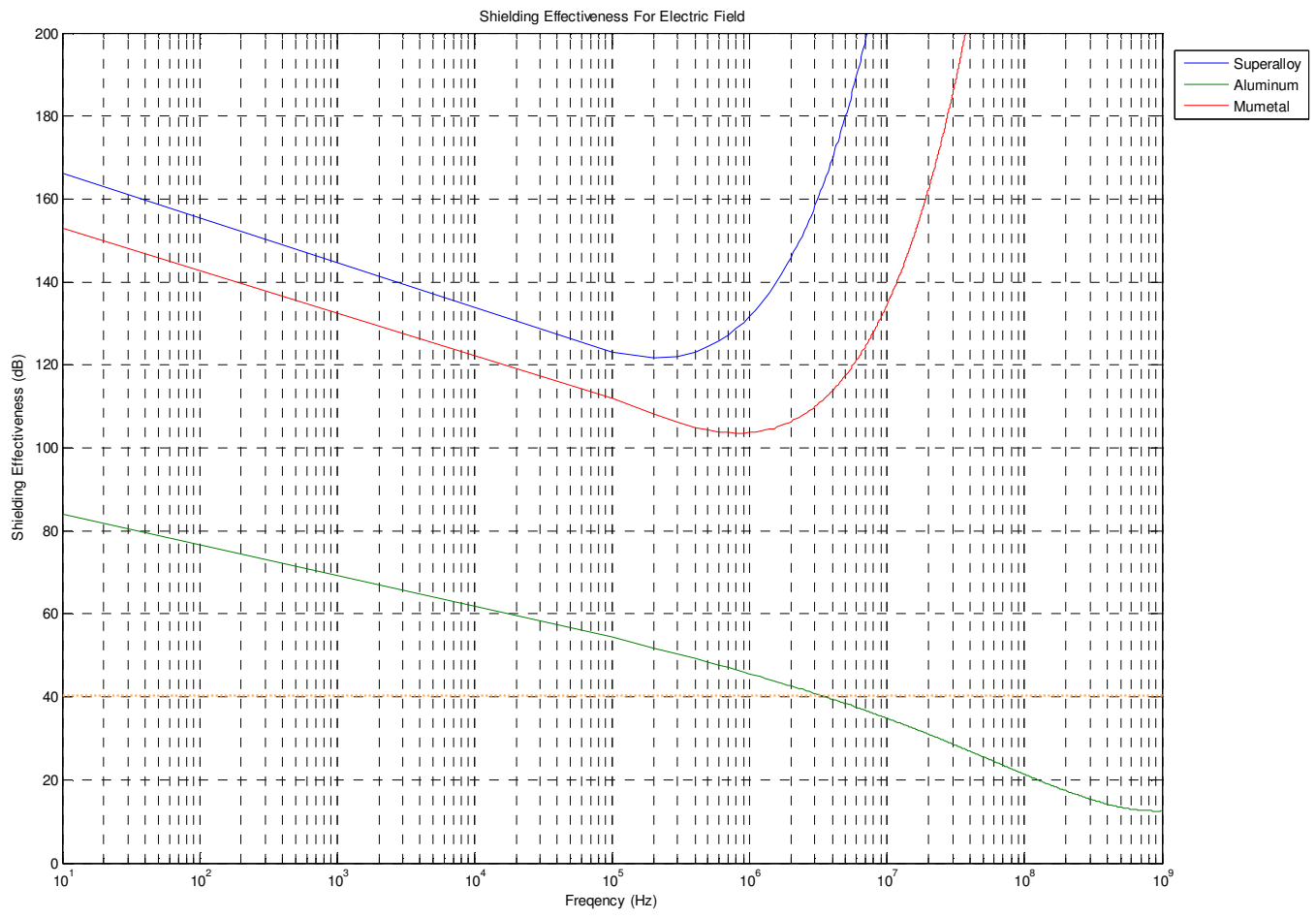

Figure 18b. Shielding Effectiveness for Electric Field Up to $200 \mathrm{~dB}$ 


\section{Plane Wave}

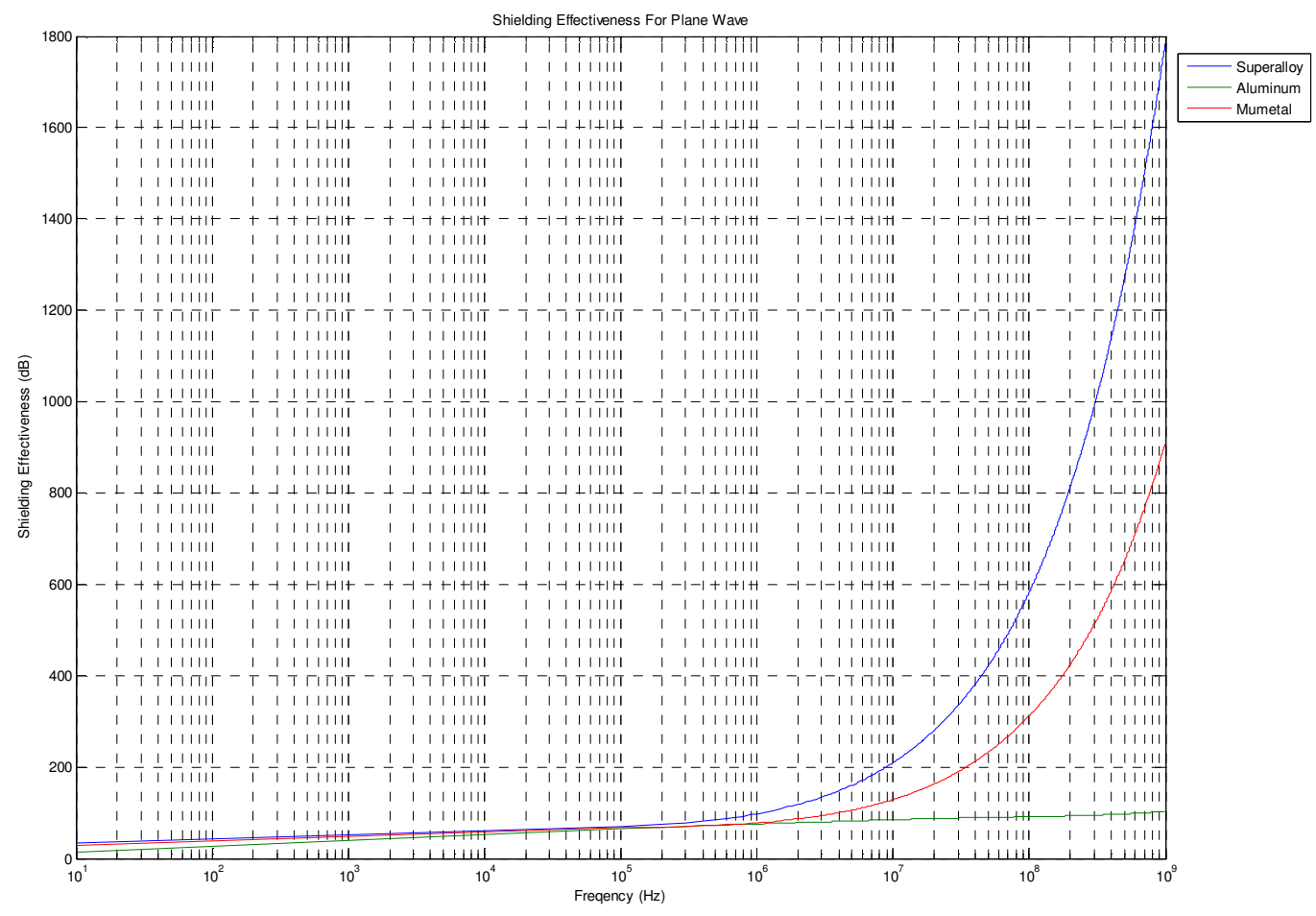

Figure 19a. Shielding Effectiveness for Plane Wave 


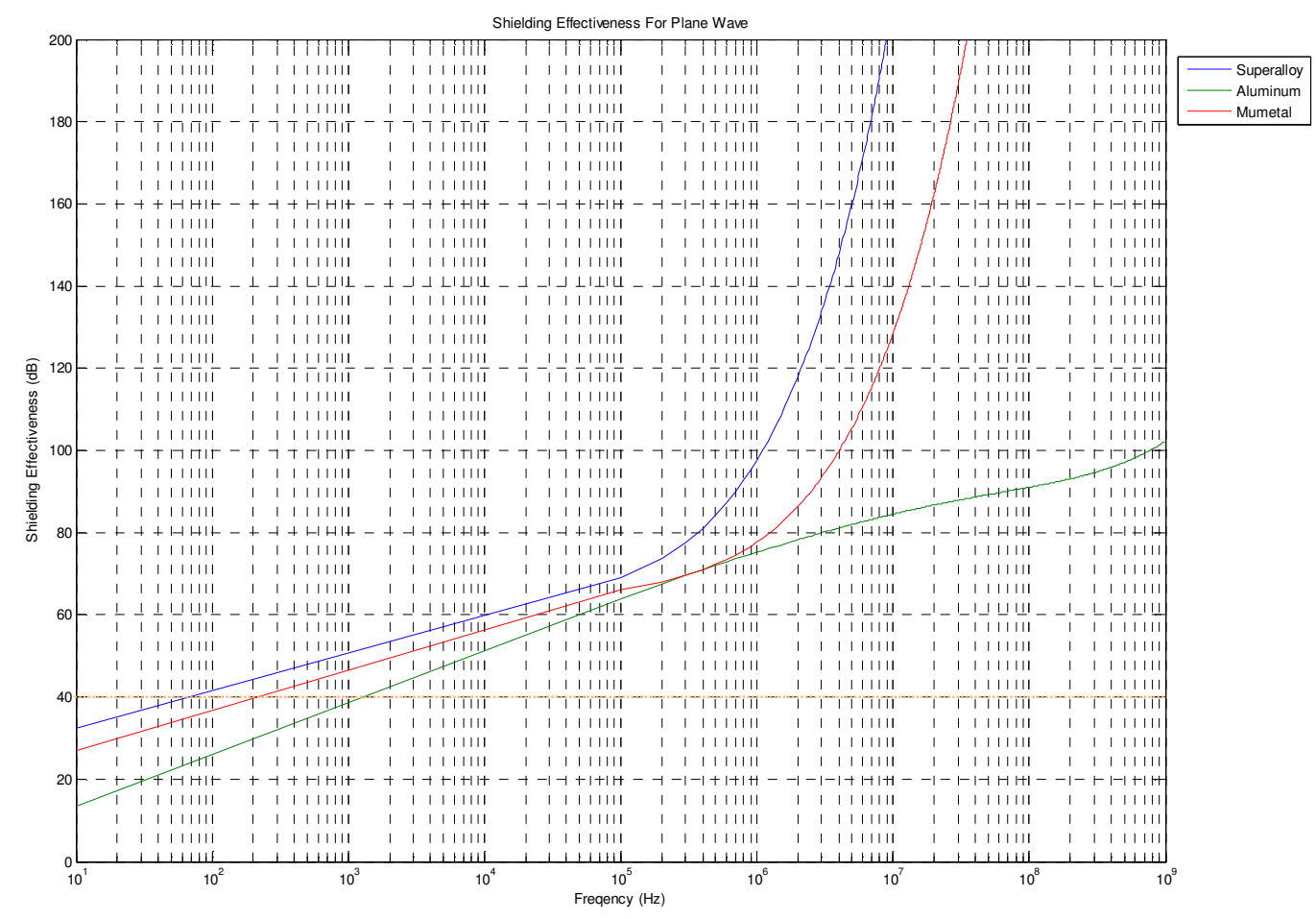

Figure 19b. Shielding Effectiveness for Plane Wave Up to $200 \mathrm{~dB}$

Tabulated results of shielding effectiveness could by observed in Table 5 of the Appendix A section.

Figures $17 \mathrm{a}$ through $19 \mathrm{~b}$ illustrates the complete shielding effectiveness for all three shielding tapes in all three fields. For all three situations, aluminum was evidently the least effective while superalloy was the most effective. Even so, aluminum is still capability of providing adequate shielding of $40 \mathrm{~dB}$ for frequencies greater than or equal to $1 \mathrm{MHz}$ in the magnetic field, less than or equal to $1 \mathrm{MHz}$ in the electric field, and greater than or equal to $5 \mathrm{KHz}$ in plane wave. 
The minimum frequency for superalloy to be sufficient in the magnetic field proved to be $5 \mathrm{KHz}$. Conversely, superalloy was efficient in the electric field for the entire frequency spectrum, and from $50 \mathrm{~Hz}$ in the plane wave.

Mumetal demonstrated results similar to superalloy. The minimum frequency for effective shielding proved to be $1 \mathrm{MHz}$ in the magnetic field, the entire frequency spectrum in the electric field, and $500 \mathrm{~Hz}$ in plane wave.

In reality, shielding tapes that provide nearly $2,000 \mathrm{~dB}$ of shielding effectiveness is unnecessary. In fact, no system to date ever required a shielding effectiveness beyond $200 \mathrm{~dB}$. Therefore, Figure 17b, 18b, and 19b show the more realistic shielding effectiveness range to be used for SGEMP fields. 


\subsection{CONCLUSIONS}

The shielding effectiveness of superalloy, aluminum, and mumetal shielding tapes satisfies the $40 \mathrm{~dB}$ shielding requirement as specified in the EMC Specifications of Military Standard Handbook 419A depending on the frequency and the SGEMP fields. Overall, the results confirmed that all three tapes were the most efficient in the electric field, which attested to being the easiest to protect against, even though aluminum was the weakest for frequencies greater than $1 \mathrm{MHz}$. Plane wave placed second in sufficiency among the fields for the selected metals. Lastly, magnetic field proved to be the most difficult to shield against for frequencies less than $1 \mathrm{MHz}$.

Despite the fact that in reality, a shielding effectiveness of $200 \mathrm{~dB}$ is well beyond satisfactory, should there ever be a situation in which shielding is needed beyond that level, a shielding effectiveness up to $2,000 \mathrm{~dB}$ can be produced from these three metals, especially superalloy and mumetal. 


\subsection{BIBLIOGRAPHY}

1. Bjorklof, Dag. "Shielding for EMC." Compliance Engineering Magazine. 1999. ETL Semko, Intertek Testing Services, Kista, Sweden. <http://www.ce-mag.com/99ARG/Bjorklof137.html>.

2. Cowdell, Robert. "New Dimensions in Shielding." IEEE Transactions on Electromagnetic Compatibility, Vol. EMC-10, No. 1, March, 1968.

3. Military Standard, MIL-HDBK-419A.

4. Paul, Clayton, R. Introduction to Electromagnetic Compatibility. New York: Wiley. 1992.

5. Rikitake, Tsuneji. Magnetic and Electromagnetic Shield. Boston, Massachusetts: D. Reidel Publishings. 1987. 
APPENDIX A-TABULATED VALUES

\begin{tabular}{|r|rrr|}
\hline & \multicolumn{3}{|c|}{ Absorption Loss } \\
\hline Frequency & Superalloy & Aluminum & Mumetal \\
\hline 10 & 0.17729 & 0.0026912 & 0.088875 \\
50 & 0.39643 & 0.0060178 & 0.19873 \\
100 & 0.56063 & 0.0085104 & 0.28105 \\
500 & 1.2536 & 0.01903 & 0.62844 \\
$1.00 \mathrm{E}+03$ & 1.7729 & 0.026912 & 0.88875 \\
$5.00 \mathrm{E}+03$ & 3.9643 & 0.060178 & 1.9873 \\
$1.00 \mathrm{E}+04$ & 5.6063 & 0.085104 & 2.8105 \\
$5.00 \mathrm{E}+04$ & 12.536 & 0.1903 & 6.2844 \\
$1.00 \mathrm{E}+05$ & 17.729 & 0.26912 & 8.8875 \\
$5.00 \mathrm{E}+05$ & 39.643 & 0.60178 & 19.873 \\
$1.00 \mathrm{E}+06$ & 56.063 & 0.85104 & 28.105 \\
$5.00 \mathrm{E}+06$ & 125.36 & 1.903 & 62.844 \\
$1.00 \mathrm{E}+07$ & 177.29 & 2.6912 & 88.875 \\
$5.00 \mathrm{E}+07$ & 396.43 & 6.0178 & 198.73 \\
$1.00 \mathrm{E}+08$ & 560.63 & 8.5104 & 281.05 \\
$5.00 \mathrm{E}+08$ & 1253.6 & 19.03 & 628.44 \\
$1.00 \mathrm{E}+09$ & 1772.9 & 26.912 & 888.75 \\
\hline
\end{tabular}

Table 1. Absorption Loss for Magnetic Field, Electric Field, and Plane Wave

\begin{tabular}{|c|c|c|c|c|c|c|c|c|c|}
\hline \multirow[b]{3}{*}{ Frequency } & \multicolumn{9}{|c|}{ Peflection Loss } \\
\hline & \multicolumn{3}{|c|}{ Magnetic Feld } & \multicolumn{3}{|c|}{ Bectric Feld } & \multicolumn{3}{|c|}{ Pane Wave } \\
\hline & Superalloy & Aluminum & Mumetal & Superalloy & Aluminum & Mumetal & Superalloy & Aluminum & Mumetal \\
\hline 10 & 18.145 & 22.059 & 10.762 & 225.62 & 289.24 & 233.6 & 91.617 & 155.24 & 99.599 \\
\hline 50 & 11.646 & 28.911 & 4.9886 & 204.65 & 268.27 & 212.63 & 84.628 & 148.25 & 92.609 \\
\hline 100 & 9.0028 & 31.889 & 2.8704 & 195.62 & 259.24 & 203.6 & 81.617 & 145.24 & 89.599 \\
\hline 500 & 3.5357 & 38.835 & -0.6016 & 174.65 & 238.27 & 182.63 & 74.628 & 138.25 & 82.609 \\
\hline $1.00 \mathrm{E}+03$ & 1.6314 & 41.835 & -1.2581 & 165.62 & 229.24 & 173.6 & 71.617 & 135.24 & 79.599 \\
\hline $5.00 \mathrm{E}+03$ & -1.106 & 8.811 & -0.47853 & 144.65 & 208.27 & 152.63 & 64.628 & 128.25 & 72.609 \\
\hline$E+04$ & -1.3654 & 51.818 & 0.78388 & 135.62 & 199.24 & 143.6 & 61.617 & 125.24 & 69.599 \\
\hline $5.00 E+04$ & 0.31412 & 58.803 & 5.2754 & 114.65 & 178.27 & 122.63 & 54.628 & 118.25 & 2.609 \\
\hline$E+05$ & 1.886 & 61.812 & 7.6709 & 105.62 & 169.24 & 113.6 & 17 & 115.24 & 9.599 \\
\hline $5.00 \mathrm{E}+05$ & 6.8594 & 68.801 & 13.831 & 84.648 & 148.27 & 92.63 & 44.628 & 108.25 & 52.609 \\
\hline $1.00 \mathrm{E}+06$ & 9.3792 & 71.811 & 16.644 & 75.617 & 139.24 & 83.599 & 41.617 & 105.24 & 49.599 \\
\hline $5.00 \mathrm{E}+06$ & 15.71 & 78.8 & 23.369 & 54.648 & 118.27 & 62.63 & 34.628 & 98.253 & 42.609 \\
\hline $1.00 \mathrm{E}+07$ & 18.563 & 81.81 & 26.317 & 45.617 & 109.24 & 53.599 & 31.617 & 95.243 & 39.599 \\
\hline $5.00 \mathrm{E}+07$ & 25.344 & 88.8 & 33.223 & 24.648 & 88.274 & 32.63 & 24.628 & 88.253 & 32.609 \\
\hline $1.00 \mathrm{E}+08$ & 28.304 & 91.81 & 36.214 & 15.617 & 79.243 & 23.599 & 21.617 & 85.243 & 29.595 \\
\hline $5.00 E+08$ & 35.228 & 98.8 & 43.177 & -5.3518 & 58.274 & 2.6296 & 14.628 & 78.253 & $22.60 \mathrm{~s}$ \\
\hline $1.00 \mathrm{E}+09$ & 38.222 & 101.81 & 46.181 & -14.383 & 49.243 & -6.4013 & 11.617 & 75.243 & 19.599 \\
\hline
\end{tabular}

Table 2. Reflection Loss for Magnetic Field, Electric Field, and Plane Wave 


\begin{tabular}{|r|rrr|rrr|rrr|}
\hline & \multicolumn{7}{|c|}{ Total Loss (without Re-Feflection Correction Factor) } \\
\cline { 2 - 9 } Frequency & \multicolumn{3}{|c|}{ Magnetic Feld } & \multicolumn{3}{c|}{ Bectric Feld } & \multicolumn{3}{c|}{ Pane Wave } \\
\cline { 2 - 9 } & Superalloy & Aluminum & Mumetal & Superalloy & Aluminum & Mumetal & Superalloy & Aluminum & Mumetal \\
\hline 10 & 18.322 & 22.062 & 10.851 & 225.79 & 289.25 & 233.69 & 91.795 & 155.25 & 99.688 \\
50 & 12.043 & 28.917 & 5.1873 & 205.04 & 268.28 & 212.83 & 85.024 & 148.26 & 92.808 \\
100 & 9.5634 & 31.897 & 3.1515 & 196.18 & 259.25 & 203.88 & 82.178 & 145.25 & 89.88 \\
500 & 4.7893 & 38.854 & 0.026843 & 175.9 & 238.29 & 183.26 & 75.881 & 138.27 & 83.237 \\
$1.00 \mathrm{E}+03$ & 3.4042 & 41.862 & -0.36934 & 167.39 & 229.27 & 174.49 & 73.39 & 135.27 & 80.487 \\
$5.00 \mathrm{E}+03$ & 2.8583 & 48.871 & 1.5088 & 148.61 & 208.33 & 154.62 & 68.592 & 128.31 & 74.596 \\
$1.00 \mathrm{E}+04$ & 4.241 & 51.903 & 3.5943 & 141.22 & 199.33 & 146.41 & 67.224 & 125.33 & 72.409 \\
$5.00 \mathrm{E}+04$ & 12.85 & 58.993 & 11.56 & 127.18 & 178.46 & 128.91 & 67.164 & 118.44 & 68.893 \\
$1.00 \mathrm{E}+05$ & 19.615 & 62.081 & 16.558 & 123.35 & 169.51 & 122.49 & 69.346 & 115.51 & 68.486 \\
$5.00 \mathrm{E}+05$ & 46.502 & 69.402 & 33.704 & 124.29 & 148.88 & 112.5 & 84.27 & 108.85 & 72.482 \\
$1.00 \mathrm{E}+06$ & 65.443 & 72.662 & 44.748 & 131.68 & 140.09 & 111.7 & 97.681 & 106.09 & 77.703 \\
$5.00 \mathrm{E}+06$ & 141.07 & 80.703 & 86.213 & 180.01 & 120.18 & 125.47 & 159.99 & 100.16 & 105.45 \\
$1.00 \mathrm{E}+07$ & 195.85 & 84.501 & 115.19 & 222.9 & 111.93 & 142.47 & 208.9 & 97.934 & 128.47 \\
$5.00 \mathrm{E}+07$ & 421.77 & 94.817 & 231.95 & 421.08 & 94.291 & 231.36 & 421.05 & 94.271 & 231.34 \\
$1.00 \mathrm{E}+08$ & 588.94 & 100.32 & 317.26 & 576.25 & 87.753 & 304.65 & 582.25 & 93.753 & 310.65 \\
$5.00 \mathrm{E}+08$ & 1288.8 & 117.83 & 671.62 & 1248.3 & 77.304 & 631.07 & 1268.2 & 97.283 & 651.05 \\
$1.00 \mathrm{E}+09$ & 1811.1 & 128.72 & 934.93 & 1758.5 & 76.155 & 882.35 & 1784.5 & 102.16 & 908.35 \\
\hline
\end{tabular}

Table 3. Total Loss for Magnetic Field, Electric Field, and Plane Wave

\begin{tabular}{|c|c|c|c|c|c|c|c|c|c|}
\hline \multirow[b]{3}{*}{ Frequency } & \multicolumn{9}{|c|}{ Re-Peflection Correction Factor } \\
\hline & \multicolumn{3}{|c|}{ Magnetic Field } & \multicolumn{3}{|c|}{ Bectric Feld } & \multicolumn{3}{|c|}{ Pane Wave } \\
\hline & Superalloy & Aluminum & Mumetal & Superalloy & Aluminum & Mumetal & Superalloy & Aluminum & Mumetal \\
\hline 10 & 37.741 & 52.839 & 26.894 & 59.459 & 141.68 & 72.735 & $\overline{59.45}$ & 141.68 & $\overline{72.72 \varepsilon}$ \\
\hline 50 & 24.157 & 67.982 & 14.915 & 44.407 & 125.71 & 57.29 & 44.399 & 125.71 & 57.283 \\
\hline 100 & 18.886 & 74.433 & 10.688 & 38.152 & 118.84 & 50.767 & 38.144 & 118.84 & 50.76 \\
\hline 500 & 8.7624 & 87.012 & 4.0344 & 24.517 & 102.93 & 36.132 & 24.51 & 102.93 & 36.126 \\
\hline $\mathrm{E}+03$ & 5.6014 & 88.615 & 2.7836 & 19.208 & 96097 & 30.159 & 9.201 & 6.096 & 0.153 \\
\hline 03 & 21 & & 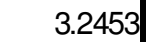 & 8.9187 & & 7.558 & 9139 & 319 & 7.553 \\
\hline & 0.93894 & & 53 & 5.6297 & & 12.909 & 5.6259 & 75 & 12.905 \\
\hline 04 & 5224 & 22 & 32 & 1.0863 & & 7204 & 1.0849 & 12 & 4.7177 \\
\hline$=+05$ & 0.14413 & 29 & 1.8116 & 33572 & 7 & 2.5045 & 0.33511 & 577 & 2.5027 \\
\hline$E+05$ & 0.001471 & 36.885 & 7592 & 0.0021715 & 96 & 0.20578 & 0.0021626 & 6.896 & .20545 \\
\hline $1.00 E+06$ & $3.76 E=05$ & 30.885 & 0.027724 & $4.95 E=05$ & 30.891 & 0.030968 & 4.92E-05 & 30.891 & 0.030895 \\
\hline $5.00 E+06$ & $5.14 \mathrm{E}-12$ & 18.176 & $9.89 \mathrm{E}-06$ & $5.83 \mathrm{E}-12$ & 18.178 & $1.04 E-05$ & $5.75 E-12$ & 18.178 & $1.03 E=05$ \\
\hline $1.00 \mathrm{E}+07$ & $5.19 \mathrm{E}-18$ & 13.458 & $2.50 \mathrm{E}-08$ & $2.28 \mathrm{E}-18$ & 13.459 & $2.60 \mathrm{E}-08$ & 1.70E-18 & 13.459 & 2.57E-08 \\
\hline$E+07$ & $4 E-40$ & 551 & $E=19$ & $5.44 E-40$ & 5.0553 & $2.69 E-19$ & $4.92 E-40$ & 5.055 & $2.60 E-19$ \\
\hline $1.00 E+08$ & $1.89 E-56$ & 2.7371 & $=-27$ & 5.04E-56 & 2.7374 & $1.58 \mathrm{E}-27$ & $3.21 E-56$ & 2.7371 & 1.48E-27 \\
\hline & $E-125$ & & $E=64$ & 2.03E-124 & & $2.65 E-62$ & 125 & & $E=6$ \\
\hline $1.00 \mathrm{E}+09$ & 6.22E-177 & 0.040759 & $E-89$ & $9.10 \mathrm{E}-177$ & 0.040863 & 9.97E-88 & $3.84 \mathrm{E}-177$ & 0.040755 & $6.88 \mathrm{E}-8$ \\
\hline
\end{tabular}

Table 4. Re-Reflection Correction Factor for Magnetic Field, Electric Field, and Plane Wave 


\begin{tabular}{|c|c|c|c|c|c|c|c|c|c|}
\hline \multirow[b]{3}{*}{ Frequency } & \multicolumn{9}{|c|}{ ShieldingEfectiveness } \\
\hline & \multicolumn{3}{|c|}{ Magnetic Feld } & \multicolumn{3}{|c|}{ Bectric Feld } & \multicolumn{3}{|c|}{ Pane Wave } \\
\hline & Superalloy & Aluminum & Mumetal & Superalloy & Aluminum & Mumetal & Siperalloy & Aluminum & Mumetal \\
\hline 10 & -19.419 & -30.777 & -16.044 & 166.34 & 83.936 & 152.97 & 32.344 & 13.562 & 26.959 \\
\hline 50 & -12.114 & -39.064 & -9.7279 & 160.64 & 78.947 & 147.56 & 40.625 & 22.552 & 35.525 \\
\hline 100 & -9.3225 & -42.536 & -7.5366 & 158.03 & 76.788 & 145.13 & 44.034 & 26.413 & 39.119 \\
\hline 500 & -3.9731 & -48.158 & -4.0075 & 151.38 & 71.741 & 139.14 & 51.371 & 35.346 & 47.111 \\
\hline $1.00 E+03$ & -2.1971 & -46.754 & -3.1529 & 148.18 & 69.548 & 136.35 & 54.189 & 39.173 & 50.335 \\
\hline $5.00 E+03$ & 1.3372 & -30.506 & -1.7365 & 139.69 & 64.389 & 129.08 & 59.678 & 47.994 & 57.043 \\
\hline $1.00 E+04$ & 3.302 & -21.239 & -0.41098 & 135.59 & 62.127 & 125.52 & 61.598 & 51.752 & 59.504 \\
\hline $5.00 E+04$ & 12.498 & 0.97176 & 8.5566 & 126.1 & 56.726 & 116.21 & 66.079 & 60.332 & 64.176 \\
\hline $1.00 E+05$ & 19.471 & 10.552 & 14.747 & 123.01 & 54.309 & 112 & 69.011 & 63.935 & 65.983 \\
\hline $5.00 E+05$ & 46.501 & 32.517 & 33.528 & 124.29 & 48.354 & 104.32 & 84.268 & 71.959 & 72.277 \\
\hline $1.00 E+06$ & 65.442 & 41.776 & 44.721 & 131.68 & 45.577 & 103.69 & 97.68 & 75.203 & 77.672 \\
\hline $5.00 E+06$ & 141.07 & 62.526 & 86.213 & 180.01 & 38.373 & 117.49 & 159.99 & 81.979 & 105.45 \\
\hline $1.00 \mathrm{E}+07$ & 195.85 & 71.043 & 115.19 & 222.9 & 34.85 & 134.49 & 208.9 & 84.475 & 128.47 \\
\hline $5.00 E+07$ & 421.77 & 89.762 & 231.95 & 421.08 & 25.611 & २23.38 & 421.05 & 89.216 & 231.34 \\
\hline $1.00 \mathrm{E}+08$ & 588.94 & 97.583 & 317.26 & 576.25 & 21.39 & 296.66 & 582.25 & 91.016 & 310.65 \\
\hline $5.00 E+08$ & 1288.8 & 117.58 & 671.62 & 1248.3 & 13.428 & 623.09 & 1268.2 & 97.033 & 651.05 \\
\hline $1.00 E+09$ & 1811.1 & 128.68 & 934.93 & 1758.5 & 12.489 & 874.36 & 1784.5 & 102.11 & 908.35 \\
\hline
\end{tabular}

Table 5. Shielding Effectiveness for Magnetic Field, Electric Field, and Plane Wave 


\subsection{APPENDIX B-EMI SHIELDING CHARACTERISTICS OF METALS}

\begin{tabular}{|c|c|c|c|c|c|c|}
\hline METAL & $\begin{array}{c}\text { SPEAIFC } \\
\text { BECTRIC } \\
\text { CONDUCTIVITY } \\
\sigma_{\mathrm{r}}\end{array}$ & $\begin{array}{c}\text { SPEARC } \\
\text { PERMEABIUTY } \\
\mu_{\mathrm{r}}(\leq 10 \mathrm{kHz})\end{array}$ & $\begin{array}{c}\text { SPEOLFC } \\
\text { ABSORPTION } \\
\text { LOSS } \\
A=k_{1} \sqrt{ } \sigma_{r} \mu_{r}\end{array}$ & 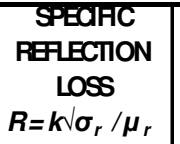 & $\begin{array}{c}\text { SPECARC } \\
\text { RERECTION } \\
\text { LOSS } \\
R(\mathrm{~dB})\end{array}$ & $\begin{array}{l}\text { DENSTY } \\
\left.\rho\left(\mathrm{g}^{\mathrm{cm}}\right)^{3}\right)\end{array}$ \\
\hline Slver & 1.064 & 1 & 1.03 & 1.3 & 0.3 & 10.501 \\
\hline Copper (solid) & 1 & 1 & 1 & 1 & 0 & 8.96 \\
\hline Copper (flame spray) & 0.1 & 1 & 0.32 & 0.32 & -10 & $\mathrm{~N} / \mathrm{A}$ \\
\hline Gold & 0.7 & 1 & 0.88 & 0.88 & -1.1 & 19.282 \\
\hline Chromium & 0.664 & 1 & 0.81 & 0.81 & -1.8 & 7.19 \\
\hline Aluminum (soft) & 0.63 & 1 & 0.78 & 0.78 & -2.1 & 2.6 \\
\hline Aluminum (tempered) & 0.4 & 1 & 0.63 & 0.63 & -4 & $\overline{\mathrm{N} / \mathrm{A}}$ \\
\hline Aluminum (household foil, 1 mil) & 0.53 & 1 & 0.73 & 0.73 & -2.8 & 2.698 \\
\hline Aluminum (flame spray) & 0.036 & 1 & 0.19 & 0.19 & -14.4 & $\mathrm{~N} / \mathrm{A}$ \\
\hline Brass (91\%an, 9\%Zn) & 0.47 & 1 & 0.69 & 0.69 & -3.3 & 8.7 \\
\hline $\operatorname{Brass}(66 \%$ a, 34\%Zn) & 0.35 & 1 & 0.52 & 0.52 & -5.7 & 8.5 \\
\hline Znc & 0.305 & 1 & 0.57 & 0.57 & -4.9 & 7.134 \\
\hline Tin & 0.151 & 1 & 0.39 & 0.39 & -8.2 & 7.287 \\
\hline Superalloy & 0.023 & 100,000 & 53.7 & 0.0005 & -65.4 & 8.9 \\
\hline 78 Permalloy & 0.108 & 8,000 & 29.4 & 0.0037 & -48.7 & 8.6 \\
\hline Purified Iron & 0.17 & 5,000 & 29.2 & 0.0058 & -44.7 & 7.85 \\
\hline Conetic AA & 0.031 & 20,000 & 28.7 & 0.0011 & -58.8 & $\overline{\mathrm{N} / \mathrm{A}}$ \\
\hline 4-79 Permalloy & 0.0314 & 20,000 & 25.1 & 0.0013 & -58 & $\overline{\mathrm{N} / \mathrm{A}}$ \\
\hline Mumetal & 0.0289 & 20,000 & 24 & 0.0012 & -58.4 & 8.75 \\
\hline Permedur(50 Qu, 1-2 V, \%Fe) & 0.247 & 800 & 14.1 & 0.0018 & -35.1 & $\mathrm{~N} / \mathrm{A}$ \\
\hline Hypernick & 0.0345 & 4,500 & 12.5 & 0.0028 & -51.1 & $\mathrm{~N} / \mathrm{A}$ \\
\hline 45 Permalloy (1200 anneal) & 0.0384 & 4,000 & 12.4 & 0.0031 & -50.2 & 8.25 \\
\hline 45 Permalloy (1050 anneal) & 0.0384 & 2,500 & 9.8 & 0.0039 & -48.1 & 8.25 \\
\hline Hot-Rolled Slicon Steel & 0.0384 & 1,500 & 7.59 & 0.0051 & -45.9 & 3.58 \\
\hline Snimax & 0.0192 & 3,000 & 7.59 & 0.0025 & -51.9 & 1.04 \\
\hline 4\%Slicon Iron (grain oriented) & 0.037 & 1,500 & 7.45 & 0.005 & -46.1 & $\mathrm{~N} / \mathrm{A}$ \\
\hline
\end{tabular}

IIIE日ectrical Insulation Magazine Nov./Dec 1990-Vol.6, No. 6

TABLE 6. EMI Shielding Characteristics of Metals 


\title{
APPENDIX C-MATLAB SOURCE CODE FOR ABSORPTION LOSS, REFLECTION LOSS, RE-REFLECTION CORRECTION FACTOR, AND SHIELDING EFFECTIVENESS
}

\author{
$\% \% \% \% \% \% \% \% \% \% \% \% \% \% \% \% \% \% \% \% \% \% \% \% \% \% \% \% \% \% \% \% \% \% \% \% \% \% \%$ \\ $\%$ Author: Cindy S Cheung \\ $\%$ Last Updated: November 10, 2008 \\ $\%$ Function: Matlab Source Code that Calculates and Plots Absorption Loss, \\ $\% \quad$ Reflection Loss, Re-Reflection Correction Factor, and \\ $\% \quad$ Shielding Effectiveness for a Superalloy, Aluminum, and \\ $\% \quad$ Mumetal Shielding 0.00035 inches thick and located 1 meter \\ $\% \quad$ from EM source \\ $\% \% \% \% \% \% \% \% \% \% \% \% \% \% \% \% \% \% \% \% \% \% \% \% \% \% \% \% \% \% \% \% \% \% \% \% \% \% \% \% \%$ \\ $\%$ Clear Output Windows \\ clear all; \\ clc; \\ $\%$ Frequency Range \\ Freq=10:10e4:1e9; \%Frequencies used for Plots \\ $\%$ Freq $=[1 \mathrm{e} 15 \mathrm{e} 1$ 1e2 5e2 1e3 5e3 1e4 5e4 1e5 5e5 1e6 5e6 1e7 5e7 1e8 5e8 1e9]; \\ $\% \% \% \% \% \% \% \% \% \% \% \% \% \% \% \% \% \% \% \% \% \% \% \% \% \% \% \% \% \% \% \% \% \% \% \% \% \%$ \\ $\%$ Absorption Loss for all Fields \\ $\% \% \% \% \% \% \% \% \% \% \% \% \% \% \% \% \% \% \% \% \% \% \% \% \% \% \% \% \% \% \% \% \% \% \% \% \% \% \%$ \\ $\mathrm{K} 1=3.34 ; \quad \%$ Constant $=131.4$ if 1 is meters; 3.34 if 1 is inches \\ $1=0.00035 ; \quad \%$ Thickness in inches \\ $\%$ Superalloy Parameters \\ SA_ur $=1 \mathrm{e} 5 ; \quad \%$ Permability \\ SA_gr $=0.023 ; \quad \%$ Conductivity \\ $\%$ Aluminum Parameters \\ Al_ur $=1 ; \quad \%$ Permability \\ Al_gr $=0.53 ; \quad \%$ Conductivity \\ $\%$ Mumetal Parameters \\ Mu_ur = 2e4; \%Permability \\ Mu_gr $=0.0289 ; \quad \%$ Conductivity \\ $\%$ Absorption Loss Equations \\ A_SA $=\mathrm{K} 1 * 1 * \operatorname{sqrt}($ Freq $*$ SA_ur $*$ SA_gr $)$; \\ A_Al $=\mathrm{K} 1 * 1 * \operatorname{sqrt}($ Freq $*$ Al_ur * Al_gr); \\ $\mathrm{A} \_\mathrm{Mu}=\mathrm{K} 1 * 1 * \operatorname{sqrt}\left(\right.$ Freq $* \mathrm{Mu} \_$ur $*$ Mu_gr);
}




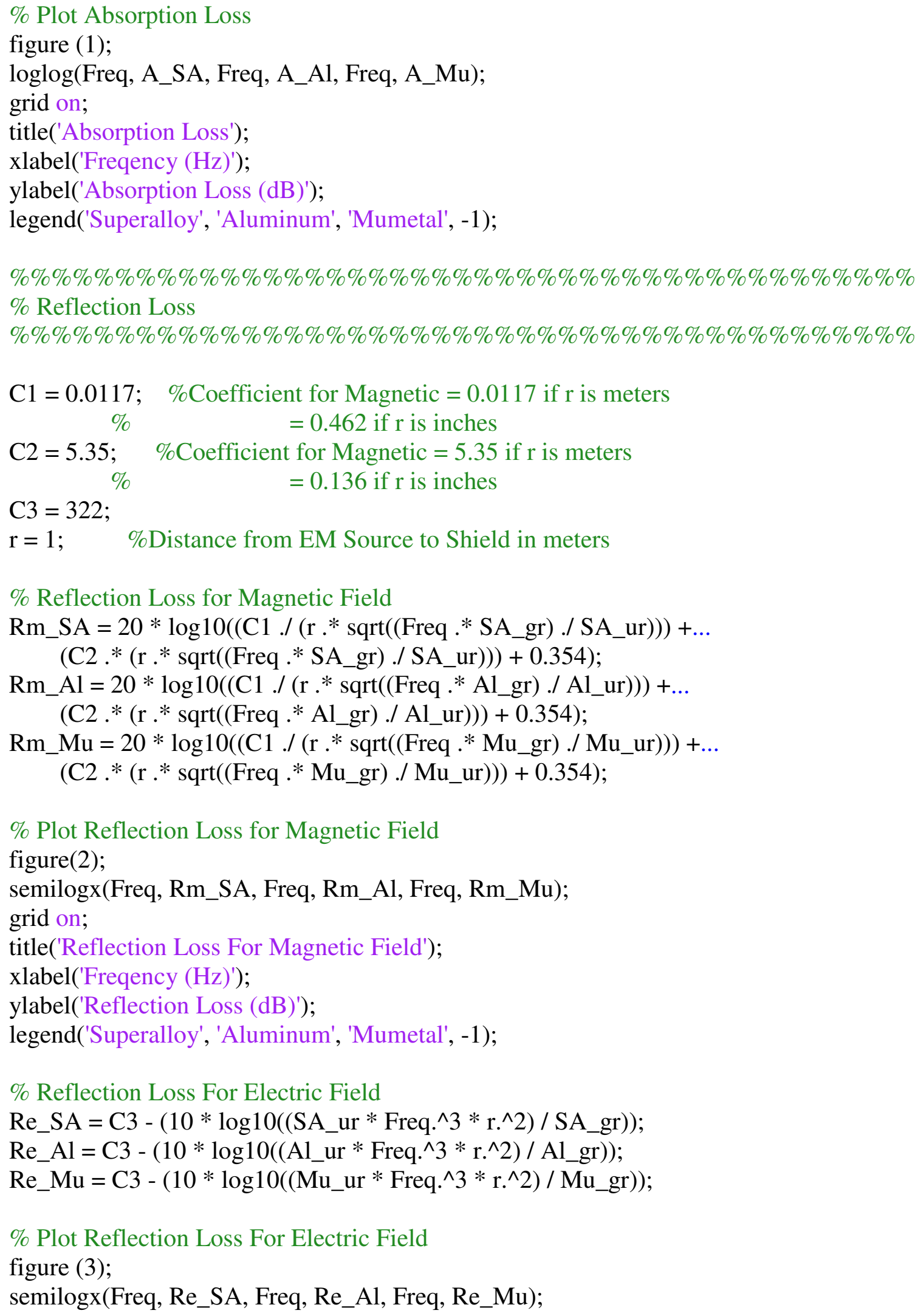


grid on;

title('Reflection Loss For Electric Field');

xlabel('Freqency $\left.(\mathrm{Hz})^{\prime}\right)$;

ylabel('Reflection Loss $\left.(\mathrm{dB})^{\prime}\right)$;

legend('Superalloy', 'Aluminum', 'Mumetal', -1);

$\%$ Reflection Loss For Plane Wave

Rp_SA $=168-20 * \log 10(\operatorname{sqrt}(($ Freq $*$ SA_ur $) /$ SA_gr $))$;

Rp_Al $=168-20 * \log 10\left(\operatorname{sqrt}\left((\right.\right.$ Freq $* A 1$ Ar $\left.\left.) / A 1 \_g r\right)\right)$;

$\mathrm{Rp} \_\mathrm{Mu}=168-20 * \log 10\left(\operatorname{sqrt}\left((\right.\right.$ Freq $*$ Mu_ur $\left.\left.) / \mathrm{Mu} \_g r\right)\right)$;

\% Plot Reflection Loss For Plane Wave

figure(4);

semilogx(Freq, Rp_SA, Freq, Rp_Al, Freq, Rp_Mu);

grid on;

title('Reflection Loss For Plane Wave');

xlabel('Freqency $\left.(\mathrm{Hz})^{\prime}\right)$;

ylabel('Reflection Loss (dB)');

legend('Superalloy', 'Aluminum', 'Mumetal', -1);

$\% \% \% \% \% \% \% \% \% \% \% \% \% \% \% \% \% \% \% \% \% \% \% \% \% \% \% \% \% \% \% \% \% \% \% \% \% \% \% \%$

$\%$ Total Loss For Magnetic Field

$\% \% \% \% \% \% \% \% \% \% \% \% \% \% \% \% \% \% \% \% \% \% \% \% \% \% \% \% \% \% \% \% \% \% \% \% \% \% \% \%$

TotalM_SA $=$ A_SA + Rm_SA;

TotalM_Al = A_Al + Rm_Al;

TotalM_Mu = A_Mu + Rm_Mu;

$\%$ Plot Total Loss For Magnetic Field

figure (5);

$\log \log ($ Freq, TotalM_SA, Freq, TotalM_Al, Freq, TotalM_Mu);

grid on;

title('Total Loss For Magnetic Field');

xlabel('Freqency $\left.(\mathrm{Hz})^{\prime}\right)$;

ylabel('Total Loss $(\mathrm{dB})$ ');

legend('Superalloy', 'Aluminum', 'Mumetal', -1);

$\% \% \% \% \% \% \% \% \% \% \% \% \% \% \% \% \% \% \% \% \% \% \% \% \% \% \% \% \% \% \% \% \% \% \% \% \% \% \% \%$

$\%$ Total Loss For Electric Field

$\% \% \% \% \% \% \% \% \% \% \% \% \% \% \% \% \% \% \% \% \% \% \% \% \% \% \% \% \% \% \% \% \% \% \% \% \% \% \% \%$

TotalE_SA $=$ A_SA + Re_SA;

TotalE_Al $=$ A_Al + Re_Al;

TotalE_Mu = A_Mu + Re_Mu;

$\%$ Plot Total Loss For Electric Field 
figure (6);

$\log \log ($ Freq, TotalE_SA, Freq, TotalE_Al, Freq, TotalE_Mu);

grid on;

title('Total Loss For Electric Field');

xlabel('Freqency $\left.(\mathrm{Hz})^{\prime}\right)$;

ylabel('Total Loss $\left.(\mathrm{dB})^{\prime}\right)$;

legend('Superalloy', 'Aluminum', 'Mumetal', -1);

$\% \% \% \% \% \% \% \% \% \% \% \% \% \% \% \% \% \% \% \% \% \% \% \% \% \% \% \% \% \% \% \% \% \% \% \% \%$

$\%$ Total Loss For Plane Wave

$\% \% \% \% \% \% \% \% \% \% \% \% \% \% \% \% \% \% \% \% \% \% \% \% \% \% \% \% \% \% \% \% \% \% \% \% \% \% \%$

TotalP_SA $=$ A_SA + Rp_SA;

TotalP_Al = A_Al + Rp_Al;

TotalP_Mu = A_Mu + Rp_Mu;

$\%$ Plot Total Loss for Plane Wave

figure(7);

loglog(Freq, TotalP_SA, Freq, TotalP_Al, Freq, TotalP_Mu);

grid on;

title('Total Loss For Plane Wave');

xlabel('Freqency $(\mathrm{Hz})$ ');

ylabel('Total Loss $(\mathrm{dB})$ ');

legend('Superalloy', 'Aluminum', 'Mumetal', -1);

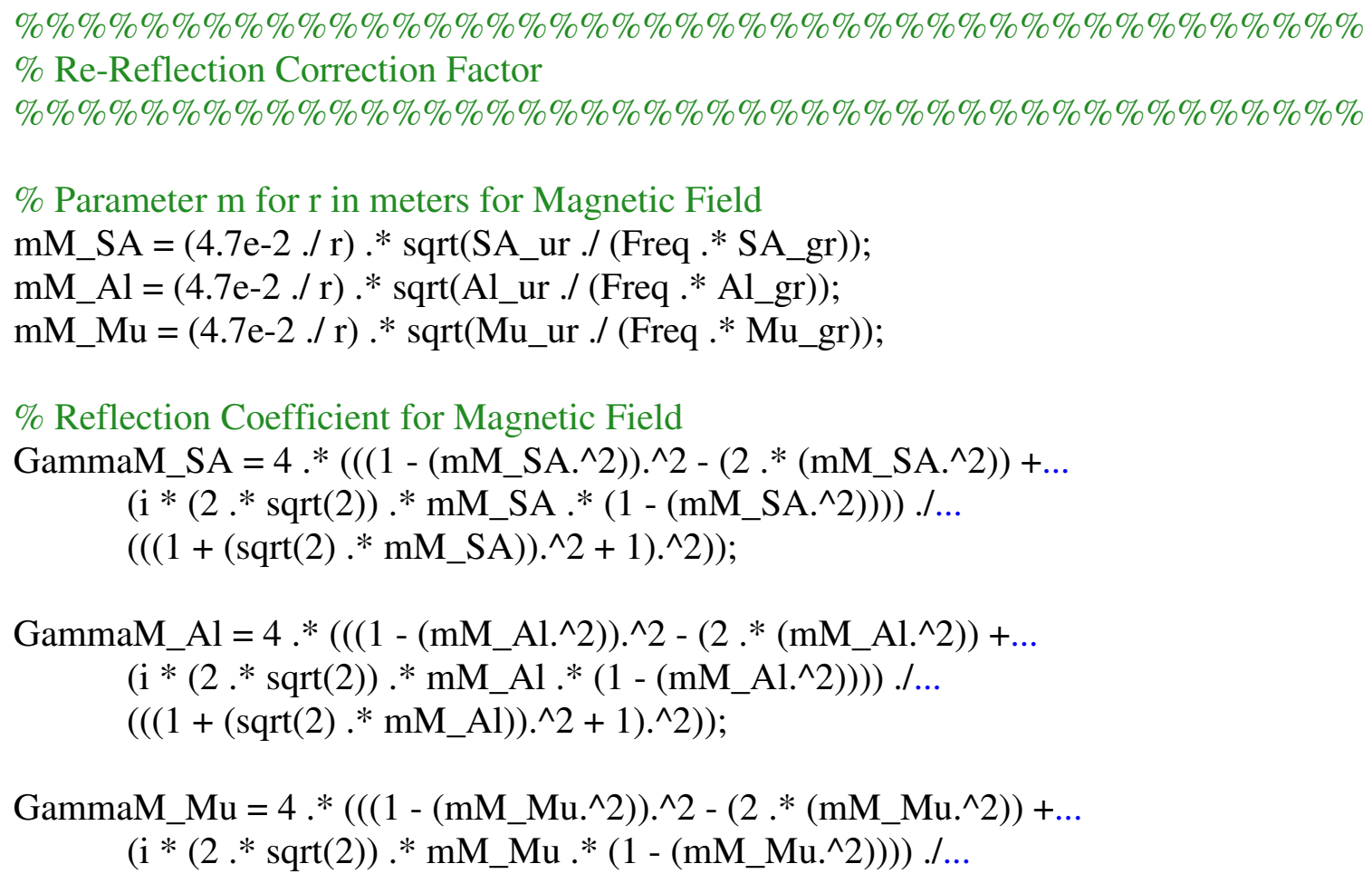




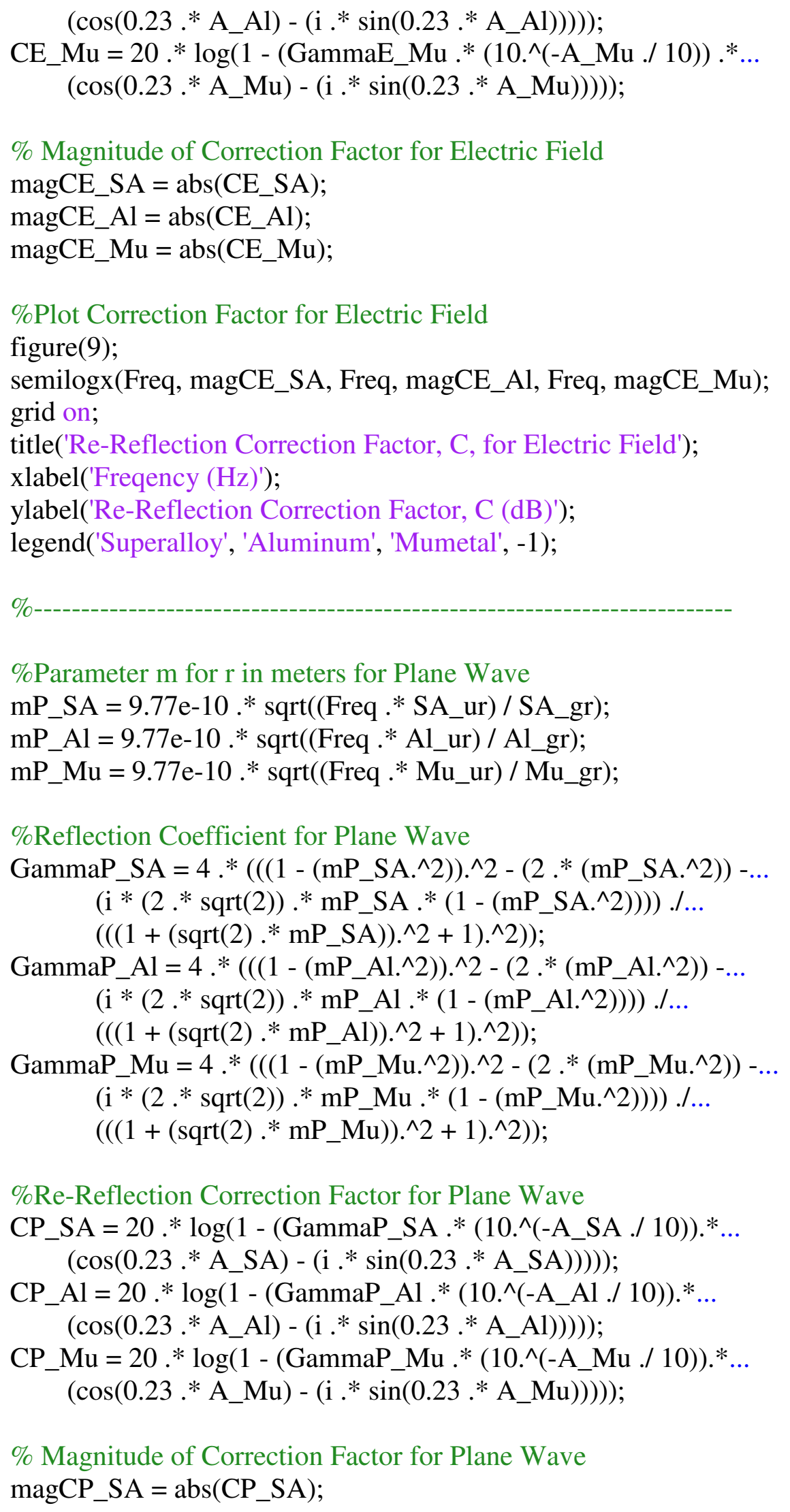




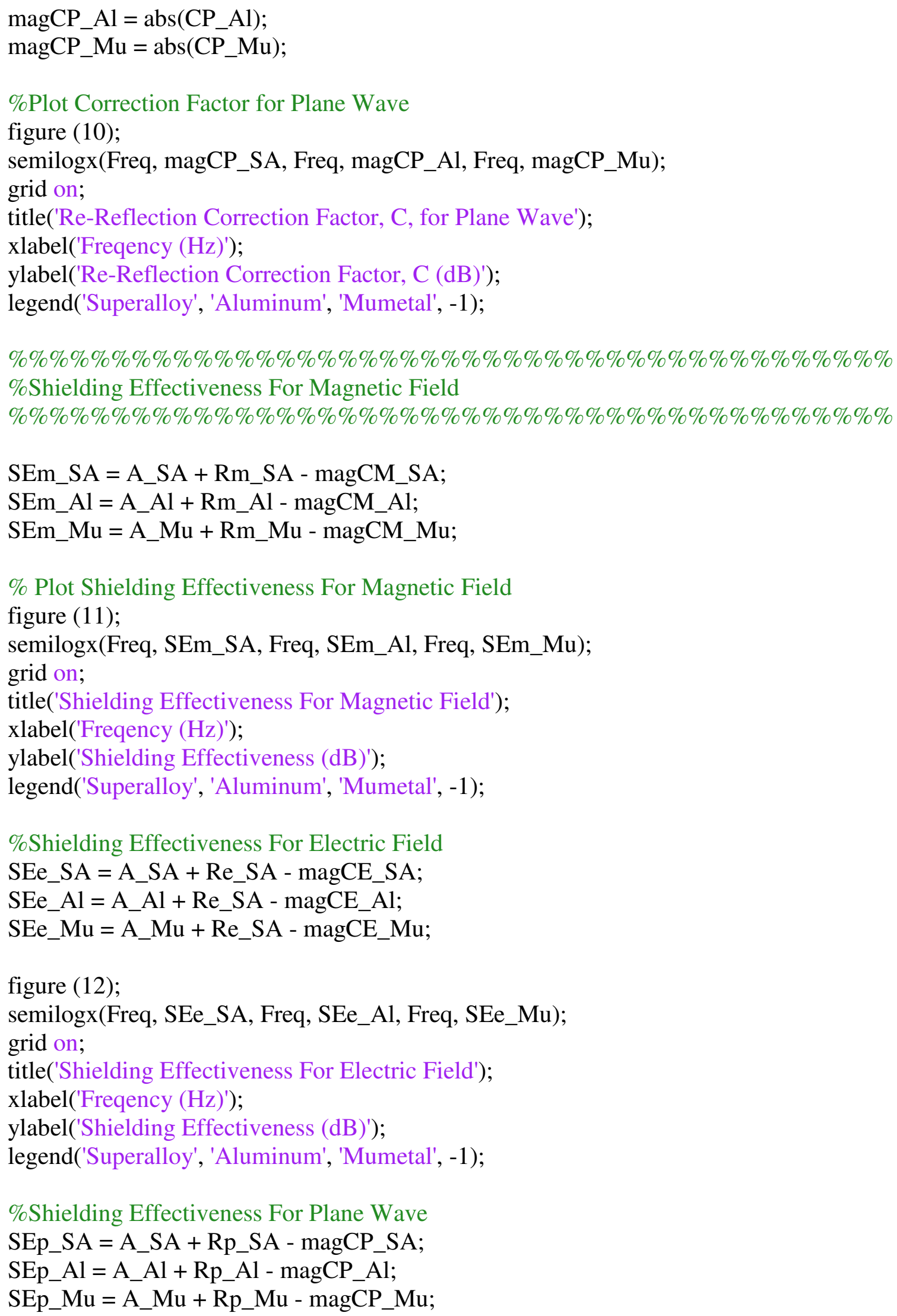


figure (13);

semilogx(Freq, SEp_SA, Freq, SEp_Al, Freq, SEp_Mu);

grid on;

title('Shielding Effectiveness For Plane Wave');

xlabel('Freqency (Hz)');

ylabel('Shielding Effectiveness (dB)');

legend('Superalloy', 'Aluminum', 'Mumetal', -1); 